Erschienen in: Stickel, Gerhard (Hrsg.): Varietäten des Deutschen. Regional- und Umgangssprachen. - Berlin, New York: de Gruyter, 1997. S. 183-220. (Institut für deutsche Sprache. Jahrbuch 1996)

\title{
JURGEN EICHHOFF
}

\section{Der „Wortatlas der deutschen Umgangssprachen": Neue Wege, neue Erkenntnisse}

\begin{abstract}
Der „Wortatlas der deutschen Umgangssprachen” war das erste großrāumig angelegte Forschungsunternehmen zur deutschen Wortgeographie, das sich von der Bindung an die polaren Varietäten Dialekt und Standardsprache freigemacht und Sprache in jenem Bereich dokumentiert hat, der die heute wichtigste Existenzform der gesprochenen Sprache darstellt: in der Umgangssprache. Im AnschluB an den Wortatlas erschienene Arbeiten werden in diesem Beitrag referiert und ergänzt, wobei Grenzbildungen in der umgangssprachlichen Lexik, aber auch in der Phonologie und Morphologie im Mittelpunkt stehen. Das Verhältnis der Umgangssprachen zu den Dialekten auf der einen und der Standardsprache auf der anderen Seite sowie die landschaftliche Synonymik bei Begriffen der modernen Welt, mit Ausblicken auf die sprachschöpferische Leistung der Umgangssprachen, bilden weitere Schwerpunkte.
\end{abstract}

Obwohl Walter Henzen schon vor fast einem halben Jahrhundert darauf hingewiesen hat, daB "für gewöhnlich von den hundert und mehr Millionen Deutschen kaum ein Drittel Mundart, sozusagen niemand die Schrift- oder Hochsprache und alle übrigen diese Zwischenstufe", nämlich "die Umgangssprache" sprechen ${ }^{1}$, hat erst die jüngere, auf die Verwendung von Sprache im sozialen Kontext zielende Linguistik diesem Bereich verstärkte Aufmerksamkeit zukommen lassen. Der Grund für die lange Vernachlässigung dieser heute zweifellos wichtigsten Erscheinungsform der gesprochenen deutschen Sprache ist nicht zuletzt der Umstand, daB das sprachliche Geschehen in diesem Bereich stark variiert. Je nach Situation und Gesprächspartner kann es auf dialektale Elemente zurückgreifen oder genuin Standardsprachliches heranziehen, ganz zu schweigen von einem zum Teil ausgesprochenen Hang zur Neuschöpfung. Umfassende Darstellungen der Varietät „Umgangssprache” haben deshalb bisher nur auf einzelörtlicher und kleinregionaler Ebene erfolgreich durchgeführt werden können. ${ }^{2}$ Allein für den Teilbereich der Lexik liegt mit dem "Wortatlas der deutschen Umgangssprachen” (WDU) eine das Ge-

1 Henzen (1954, S. 19f.); „Deutsche” hat Henzen gewiß im Sinne von „Deutschsprachige” gemeint.

2 Literaturangaben bei Munske (1983, S. 1002). Siehe jetzt auch Kallmeyer (1994). 
samtgebiet der deutschen Sprache weitgehend übergreifende Dokumentation vor.

Auf der Grundlage des WDU sind mehrere Arbeiten erschienen, die umgangssprachliche Erscheinungen teils in neue historische Zusammenhänge einordnen, teils sie für die Aufdeckung von Entwicklungstendenzen im Bereich der Gegenwartssprache fruchtbar machen. Diese Arbeiten werden im folgenden referiert und ergänzt, wobei es hauptsächlich um Grenzbildungen in der umgangssprachlichen Lexik, aber auch in der Phonologie und Morphologie geht. Weitere Kapitel gelten dem Verhältnis der umgangssprachlichen Lexik zur Lexik der Dialekte auf der einen, der Standardsprache auf der anderen Seite. DaB lexikalische Varianten nicht nur aus alten Zeiten auf uns gekommen sind, sondern auch heute noch entstehen, zeigt das abschlieBende Kapitel. Einleitend wird das umgangssprachliche Stufenmodell präzisiert, das im ersten Band des WDU verwendet worden ist, um die Aufgliederung des umgangssprachlichen Bereichs in regionale Varietäten zu illustrieren.

\section{Das umgangssprachliche Stufenmodell}

Der Terminus Umgangssprache wird in der Sprachwissenschaft mit unterschiedlichen Bedeutungen verwendet. ${ }^{3}$ Im Zusammenhang unserer Darstellung verstehen wir unter Umgangssprachen (unter bewußter Verwendung des Plurals) zunächst regionaltypische sprachliche Varietäten, die je nach Region in einem spezifischen Bereich des Spektrums zwischen den kleinräumig gegliederten Dialekten und der übergreifenden Standardsprache ihren Platz haben. An welcher Stelle dieses Spektrums die jeweilige regionale Umgangssprache lokalisiert ist, $d . h$., ob sie diesen oder jener nähersteht, beruht auf historischen Entwicklungen. Im Norden des deutschen Sprachgebiets sind die Umgangssprachen aus der Vorform der heutigen Standardsprache, der neuhochdeutschen Schriftsprache, entstanden, die hier zunächst als geschriebene Sprache Aufnahme fand, dann aber auch mündlich in formellen Situationen und als Mittel der überregionalen Kommunikation das Niederdeutsche mehr und mehr ablöste. Das Niederdeutsche spielt heute nur noch als Dialekt eine bescheidene und weiter abnehmende Rolle. Als sprachliches Substrat macht es sich aber in den norddeutschen Umgangssprachen durch die Färbung bestimmter Vokale, in der Realisierung bestimmter Konsonantenverbindungen, gelegentlich in der Syntax und nicht zuletzt im Wortschatz geltend. In Süddeutschland sind die Umgangssprachen dagegen nicht

3 Zur Forschungslage siehe Munske (1983, S. 1002); zur Forschungsgeschichte Bichel (1973) und Radtke (1973). 
"gesunkene Hochsprache." ${ }^{4}$ Vielmehr leiten sich hier die städtischen Umgangssprachen von Dialekten her, die in ihrem Umland vielfach noch lebendig sind. Der Unterschied zu den Dialekten ist ein gradueller, nicht wie im Norden ein qualitativer. In den Städten Öster reichs ist die Situation nach Wiesinger ${ }^{5}$ "diffizil". Neben den Dialekten (vor allem in der Form der moderneren "Verkehrsdialekte") wird von der jüngeren Generation und in den Städten mehr und mehr "Umgangssprache" verwendet. ${ }^{6}$ Darunter versteht man in Österreich ein "Ausgleichsprodukt" $z$ wischen den Dialekten und der Standardsprache, das allerdings der Standardsprache nähersteht und von den Dialekten durch einen ${ }_{n}$ auffallige[n] Einschnitt" getrennt ist. ${ }^{7}$ Insofern kann man von einer Situation ähnlich der in Norddeutschland sprechen, wobei den unterschiedlichen Ausprägungen der Umgangssprache dann im wesentlichen nur die jeweils unterschiedlichen dialektalen Einflüsse zugrunde lägen. In den Städten der Schweiz, Südtirols und Luxemburgs werden dagegen im täglichen Umgang uneingeschränkt Sprachformen verwendet, die sich nicht als distinkte sprachliche Schicht selbständig gemacht haben. Ihnen steht nicht nur nach dem Verständnis der Sprecher, sondern auch bei Anlegung wissenschaftlicher Maßstäbe die Bezeichnung "Dialekt" 8 zu. Umgangssprachen im Sinne einer Definition, die von einer $Z$ wischenform zwischen den Dialekten und der Standardsprache ausgehen, gibt es hier folglich nicht.

4 Moser (1960, S. 227 u. 231). Nach den obigen Ausführungen wäre genauer von "gesunkener Schriftsprache" zu sprechen.

5 Wiesinger (1980, S. 181).

6 Wiesinger (1980, S. 183). Einer Umfrage des Germanistischen Instituts der Universität Wien vom Jahre 1984-85 zufolge verwendet die Bevölkerung der Mittelschicht je etwa zur Hälfte Dialekt und „Umgangssprache”; in mittleren und grỏßeren Städten war das Verhältnis sogar 63 Prozent "Umgangssprache” bei nur 32 Prozent Dialekt (und 5 Prozent Standardsprache): Wiesinger (1989, S. 445-47).

7 Wiesinger 1980, S. 183.

8 Strukturlinguistisch definiert von Goossens (1977, S. 21) als „der als Ausdrucksweise eines Ortes zu betrachtende, auf lokale Verwendung zielende Komplex von Sprechweisen, bei dem zur Aufhebung der Differenzen zum hochsprachlichen System, im Vergleich zu den anderen am gleichen Ort vorkommenden Sprechweisen dieser Sprachgemeinschaft, eine maximale Anzahl von Regeln notwendig ist." In Luxemburg ist der Dialekt inzwischen in den Stand einer "indominanten Nationalsprache" erhoben worden (Hoffmann 1979, S. 134; vgl. auch S. 41ff.). 
Die von Nord nach Süd gestufte Abfolge der regionaltypischen Umgangssprachen ist in Abbildung 1 veranschaulicht. In diesem Modell, das zunächst für phonologische und morphologische Erscheinungen gilt, wird u.a. sichtbar, daB im Norden die Stufen großräumiger sind als im Süden, wo sich die Kleinräumigkeit der Dialekte in den aus ihnen hervorgegangenen Umgangssprachen widerspiegelt.

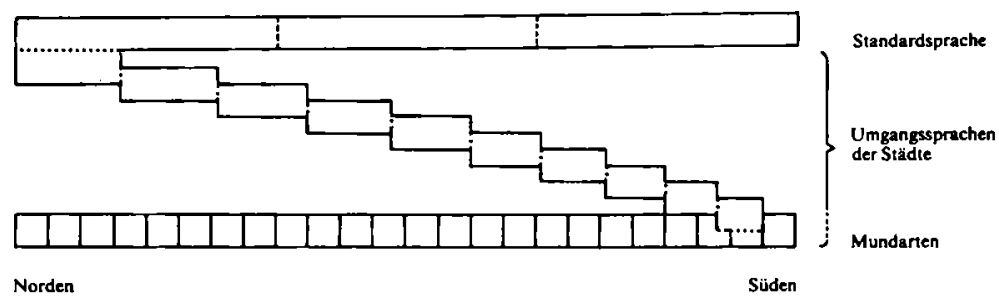

Abb. 1: Schematische Anordnung der Umgangssprachen zwischen Standardsprache und Mundarten.

Die Abbildung unterscheidet sich in einem nicht unwesentlichen Punkt von jener, die in der Einleitung zum ersten Band des WDU abgedruckt ist. Dort ist ein Abstand angedeutet $\mathbf{z w i s c h e n ~ d e r ~ S t u f e ~ d e r ~ n o r d d e u t - ~}$ schen Umgangssprache, die der Standardsprache am nächsten steht, und dieser selbst. Inzwischen ist der Trend zum alltäglichen Gebrauch standardnäherer Sprachformen fortgeschritten. Wichtiger noch: mit dem Begriff "Standardsprache" ist die strenge Norm, die ehemals mit dem Begriff "Hochsprache" sich verband, gelockert worden. Die Aussprachenorm der Standardssprache ist zur "Gebrauchsnorm" geworden und orientiert sich nicht mehr an den Bedürfnissen von Bühne und Rednerpult, sondern an der "Sprechwirklichkeit." Im Bereich der Lexik haben die standardsprachlichen Wörterbücher mehr und mehr (auch unter Auswertung der Karten des WDU) regionales Wortgut aufgenommen und damit dem Prinzip eines regionalen Standards zur Anerkennung verholfen. Für eine nicht geringe Zahl von Sprechern ist die Standardsprache bereits gewohnheitsmäBig die Sprache des täglichen Umgangs geworden, vor allem in Norddeutschland. Der kollektive Sprachgebrauch des täglichen Umgangs ${ }^{10}$ liegt aber auch hier unterhalb der standardsprachlichen Normen. Die revidierte Abbildung sucht der Entwicklung gerecht

9 Vorwort der Dudenredaktion zu Mangold (1990, S. 5).

10 „quasi die Schnittmenge der sprachlichen Realisierungen in alltāglichen Situationen" nach Friebertshäuser/Dingeldein (1985, S. 45). 
zu werden, indem sie die Bereiche "norddeutsche Umgangssprache" und "Standardsprache" aneinanderrückt und durch die Punktierung der trennenden Linie das Bestehen zahlreicher Gemeinsamkeiten und flieBender Übergänge andeutet. Auch die Übergänge zwischen den einzelnen Stufen der regionaltypischen Umgangssprachen sind durch punktierte Linien markiert. Damit ist ausgedrückt, daB es sich bei den Umgangssprachen nicht um scharf voneinander abgehobene Varietäten handelt, sondern eher um ein gestuftes Kontinuum. Für Österreich stellt unsere Skizze den insgesamt (noch) überwiegenden Sprachgebrauch, die Verwendung des Dialekts, dar und reflektiert nicht die Umbrüche, die sich im Sprachgebrauch der jüngeren Generation und in den Städten abzeichnen.

\section{Der, Wortatlas der deutschen Umgangssprachen”}

Der "Wortatlas der deutschen Umgangssprachen” beruht auf einer umfassenden Erhebung, die die Bundesrepublik Deutschland in den Grenzen von 1949-1989, die damals noch bestehende Deutsche Demokratische Republik, Österreich sowie die deutschsprachigen Teile der Schweiz und Italiens (Südtirol) einschloB. Eine erste Befragung während der Jahre 1971-1976 umfaBte 402 Orte und resultierte in den zwei ersten Bänden ${ }^{11}$ mit insgesamt 108 Wortkarten. Eine anschlieBende zweite Umfrage, durchgeführt im wesentlichen in den Jahren 1977-1987, fügte einen Ort in Südtirol sowie die Stadt Luxemburg hinzu und erbrachte Material für zwei weitere Bände, von denen der erste mit 62 Wortkarten im Jahre $1993^{12}$ publiziert wurde. Ein abschlieBender vierter Band, der voraussichtlich 57 Wortkarten enthalten wird, erscheint 1997. Der Band 2 enthält einen Anhang von 17, der Band 4 einen von voraussichtlich 22 Karten, auf denen die areale Verbreitung phonologischer, morphologischer und syntaktischer Varianten in den Umgangssprachen dargestellt ist.

11 Eichhoff (1977), Eichhoff (1978). - Die Karten der beiden ersten Bände sind über die Bandgrenze hinweg fortlaufend numeriert. In den Bānden 3 und 4 wird jeweils eine neue Numerierung angefangen; die Karten sind dann mit „Bandzahl - Kartennummer” (z.B. 3-12 für Karte 12 im dritten Band) identifiziert. Diese Methode wird im folgenden für die beiden ersten Bände nachträglich eingeführt. Die erste Zahl gibt also jeweils die Bandzahl, die zweite die Kartennummer an, auch bei Band 2, wo die Zählung der Karten erst mit 55 beginnt.

Eichhoff (1993). 
Ziel der Erhebung war es, den im deutschen Sprachgebiet in alltäglichen Situationen üblicherweise verwendeten Wortschatz zu erfassen. ${ }^{13}$ In Anbetracht der skizzierten geographischen Stufung der in alltäglichen Situationen üblicherweise gesprochenen Varietäten galt es, einen Bruch zu voliziehen zu den hergebrachten Ansätzen wortgeographischer Forschung, die sich ausschlieblich auf die polaren Varietäten des Deutschen, die Dialekte auf der einen und die Standardsprache auf der anderen Seite bezogen hatte. ${ }^{14}$ Man war davon ausgegangen, dab die sprachliche Varietät konstant gehalten werden müsse, damit die Variable "Raum" die gewünschten landschaftlichen Varianten (Heteronyme) hervorbringen konnte. Der Forschungsansatz des WDU ist demgegenüber ein pragmalinguistischer. Selbstverständlich ist auch hier der räumliche Aspekt variabel. Als Konstante ist jedoch die sprachliche Verwendungsweise („Sprache im täglichen Umgang”) gesetzt. Um Elemente welcher Varietät es sich bei dem erhobenen Wortgut dann handelt, ist erst sekundär von Bedeutung und gegebenenfalls durch linguistische Kriterien zu ermitteln. Bei groBräumigen Erhebungen wie dieser werden die Elemente sprachlicher Verwendungsweise mit großer Wahrscheinlichkeit unterschiedlichen sprachlichen Varietäten angehören. ${ }^{15}$ Bei unserer

13 Bei der Konzeption des Projekts spielten u.a. die Nöte des Deutschunterrichts im Ausland eine Rolle. Wer im Ausland Deutsch gelernt hat, trifft im deutschen Sprachgebiet regionalen Wortgebrauch an, auf den der Unterricht nicht vorbereitet hat und den der AuBenstehende auch nicht wie jemand, der im deutschen Sprachgebiet aufgewachsen ist, als regional oder stilistisch motiviert zu interpretieren vermag. Auch in der deutschsprachigen Literatur treten die regionalen Varianten für den Deutschlernenden oft irritierend in Erscheinung (Eichhoff 1972), wobei die Wörterbücher nur bruchstückhafte und auf veralteten oder ungeeigneten Quellen (z.B. den Dialekten) beruhende Hilfe boten (Niebaum 1984, Eichhoff 1988).

14 Kretschmer (1918), Mitzka/Schmitt (1951-80). Die Beschränkung war durch das Forschungsinteresse der Exploratoren motiviert. Mitzka ging es vor allem um sprachhistorisch auswertbares Material, das in den altertümlicheren Dialektformen an ehesten bewahrt ist. Kretschmer sah seine Aufgabe in der interpretierenden Bestandsaufnahme mit dem Ziel zu zeigen, daB „die hochdeutsche Gemeinsprache [...] im Wortschatz nicht zur vollen Einheitlichkeit vorgedrungen" (S. 2) ist. Beiden Unternehmungen ist der Nachteil gemein, daß die Varietāten, aus denen sie ihr Material schōpften, in weiten Bereichen des Untersuchungsgebiets nur von einem geringeren Anteil der Gesamtbevölkerung in alltäglichen Situationen tatsächlich verwendet wurden.

15 Vgl. Dingeldein (1991, S. 110-11). 


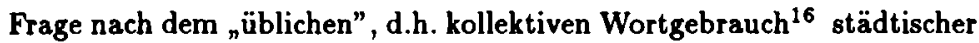
Bevölkerungen jüngeren Alters darf davon ausgegangen werden, daB im Gebiet der heutigen Bundesrepublik der Wortgebrauch in der jeweiligen Varietät "Umgangssprache" erhoben worden ist. In der Schweiz und in Südtirol, zum Teil auch in Österreich, handelt es sich dagegen um den Wortgebrauch in der Varietät "Dialekt" ${ }^{17}$.

Schon Walter Henzen ${ }^{18}$ ist zu der Erkenntnis gelangt, daB sich die Umgangssprachen ${ }_{n}$ am leichtesten [...] umschreiben, aber am schwersten [...] beschreiben" lassen. Das gilt auch weiterhin. Die dargestellte horizontale (geographische, diatopische) Gliederung kreuzt sich mit vielfältigen Faktoren, die im aktuellen Sprachgebrauch vertikal gerichtet sind und zur Bildung von Ebenen und Nischen innerhalb der Umgangssprachen führen. $\mathrm{Zu}$ diesen Faktoren gehören vor allem der Gebrauch der Sprache in unterschiedlichen soziokulturellen Gruppen (diastratische Faktoren), in unterschiedlichen Generationen von Sprechern (diaphasische Faktoren) und in unterschiedlich gewerteten sozialen Interaktionen (diasituative Faktoren). Jedoch sind davon eher die lautlichen, weniger die den Gegenstand des Atlasprojekts bildenden lexikalischen Komponenten der Sprache betroffen. Lautliche Erscheinungen werden auf den Karten des WDU im allgemeinen lediglich in linguistisch markanten und geographisch relevanten Fällen berücksichtigt. Gegenstand des Darstellungsinteresses sind sie nur auf den Karten der Anhänge in den Bänden 2 und 4.

\section{Nord/Süd-Gliederung in der umgangssprachlichen Lexik}

Bei einer groBen Zahl der Karten des WDU springt ins Auge, daB das deutsche Sprachgebiet sich in einen nördlichen und einen südlichen Bereich teilt, wobei jeder Bereich durch ein oder mehrere landschaftliche Synonyme (Heteronyme) repräsentiert sein kann. Von diesem Befund ausgehend, bezeichnen Munske (1983) und Durrell (1989) die bisherige,

16 Die Gewährspersonen wurden bewuft nach dem üblichen Sprachgebrauch am Ort, nicht nach ihrem eigenen Sprachgebrauch gefragt.

17 Der Terminus Umgangssprachen im Titel des WDU ist also im Sinne von 'Sprachverwendungsweise' zu verstehen. $V_{g l}$. auch Bichel (1973 passim, bes. S. 365-69). - Dingeldein (1991, bes. S. 38) hat die Verwendung der Bezeichnung Umgangssprache sowohl für eine sprachliche Varietät wie auch als Verwendungsweise mit guten Gründen bemängelt; jedoch gibt die von ihm im AnschluB an Porzig (s. Bichel 1973, S. 368) für letztere gewählte Bezeichung Alltagssprache zu neuen Mißverständnissen Anlaß.

Henzen (1954, S. 21). 
auf Dialektunterschieden beruhende Gliederung des Deutschen in die Bereiche Niederdeutsch, Mitteldeutsch und Oberdeutsch als für das SprachbewuBtsein der Gegenwart nicht mehr relevant. Während Munske als erster die groBräumige Zweiteilung des Sprachgebiets im Bereich der Umgangssprachen in die Diskussion einbringt, stellt Durrell sie in größere, das SprachbewuBtsein und die sprachlichen Entwicklungstendenzen einbeziehende Zusammenhänge. Insgesamt wird damit die Raumteilung des Deutschen auf neue Grundlagen gestellt. Munske ${ }^{19}$ diagnostiziert eine Nord/Süd-Gliederung bei mehr als der Hälfte der 54 Karten des ersten Bandes. ${ }^{20}$ Indem er die auf den Punkt-Symbolkarten erkennbaren Flächen gleichen Wortgebrauchs durch Linien voneinder scheidet, macht er die "dominierende Zweiteilung" des deutschen Sprachgebiets mit Hilfe einer kombinierten Isoglossenkarte sichtbar. ${ }^{21}$ Auf ihr vereinigen sich 16 Isoglossen zwischen den Städten Hof im Osten und Fulda im Westen zu einem Bündel, das sich dann allerdings nach Nordwesten und nach Südwesten auffächert. Munske schlieBt seinen Beitrag mit der Feststellung: „Aufgrund der verbreiteten Kenntnis diatopischer Varianten der umgangssprachlichen Lexik (wegen ihrer Verwendung in der überregionalen Kommunikation) ist deren regionaler Symptomwert erheblich größer, als dies für dialektale Lexik gilt. Dem umgangssprachlichen Wortschatz kommt deshalb vergleichsweise größere Bedeutung für die Konstitution umgangssprachlicher Sprachräume zu." ${ }^{22}$

Durrell setzt die Scheide zwischen dem nördlichen und dem südlichen umgangssprachlichen Areal, die in der Nähe des Mains verläuft, mit der hergebrachten Vorstellung einer die deutsche Kulturlandschaft durchzie-

19 Munske (1983, S. 1013-15).

20 Nur der erste Band lag Munske zur Zeit der Abfassung seines Beitrags vor.

21 Munske (1983, S. 1014). Sowohl Munske wie dann auch Durrell (1989, S. 102) sind sich der Problematik des Vorgehens bewuBt. Den Karten des WDU wird hier eine Funktion aufgebürdet, die zu übernehmen sie von den methodischen Voraussetzungen ihrer Erarbeitung her nicht geschaffen worden sind. Die örtlichen Eintragungen, die sich auf Angaben von jeweils nur ein oder zwei Gewährspersonen stützen, können nicht in jedem einzelnen Fall hundertprozentig zuverlässig sein. Die Karten sind flächengetreu, das heiBt, sie erlauben das Identifizieren von Arealen gleichen Wortgebrauchs. Das Ziehen von Isoglossen geht jedoch von der Annahme ortsgetreuen Belegmaterials aus. Die im folgenden abgebildeten Karten, seien es Isoglossenkarten oder die auf Isoglossenbündeln beruhenden Wabenkarten, sind deshalb nur mit dem Vorbehalt zu sehen, daB die Menge der Belege etwaige Ungenauigkeiten in Einzelfällen relativiert.

Munske (1983, S. 1015). 
henden sog. "Mainlinie" in Beziehung. In dieser Bezeichnung verfestigt sich der Umstand, daB in der mythologischen Geographie Deutschlands [...] ungefähr im Bereich des Maintals eine Grenze wahrgenommen wird zwischen Gebieten, die ein Nord- bzw. Süddeutscher in schwerlich näher zu spezifizierender Hinsicht als 'heimisch' bzw. 'fremd' empfindet." ${ }^{23}$ Als Hintergrund sieht Durrell die politischen Konstellationen des 19. Jahrhunderts, in dem jahrzehntelang die preußischen und österreichischen Interessen in diesem Gebiet aufeinanderstießen, bis schließlich von 1866 bis 1871 die Südgrenze Sachsens, Thüringens, des preußisch gewordenen Nassau und der Rheinprovinz zugleich die Südgrenze des von Preußen beherrschten Norddeutschen Bundes wurde.

Durrell stellt die Frage, ob sich für eine so ausgeprägte Vorstellung von Gegensätzen, wie sie sich in dem Begriff "Mainlinie” niedergeschlagen hat, nicht auch in der Sprache eine Stütze finden müsse. In der mundartlichen Lautgeographie, die die Basis der traditionellen Einteilung in Mundarträume bildet, läßt sich an dieser Stelle eine signifikante Grenze jedoch nicht festellen. Zwar verläuft in der Nähe des Mains die Lautverschiebungsgrenze, die in dem Wort $A$ pfel unverschobenes $p$ im Norden von $p f$ im Süden trennt. Sie liegt im Osten innerhalb des Thüringischen ein beträchtliches Stück nördlich des Mains, um den FluB nach dem zweiten Drittel seines Verlaufs in Nord/Süd-Richtung zu überqueren, bevor sie in wiederum eher ost/westlicher Richtung nördlich von Karlsruhe den Rhein erreicht. Es ist die südlichste der Verschiebungslinien, sieht man von $k / k c h$ im Alemannischen ab. In der Sprachwissenschaft ist ihr deshalb ein besonderer Status zugesprochen worden als Trennungslinie zwischen dem "Oberdeutsch" genannten Raum im Süden, wo die Lautverschiebung voll durchgeführt wurde, und der nördlich sich anschließenden „mitteldeutschen” Übergangszone. Detailuntersuchungen haben jedoch gezeigt, daß diese Grenze im Gefüge der mundartlichen Raumgliederung recht isoliert dasteht. Durrell sieht sich ${ }^{2} \mathrm{zu}$ dem SchluB gezwungen, $\mathrm{daB}$ für die Raumgliederung der deutschen Mundarten die Mainlinie unbedeutend ist." ${ }^{24}$ Die wesentlichen mundartlichen Lautgrenzen liegen beträchtlich weiter nördlich, vor allem die für die Mundartgliederung bedeutendste, die $i k / i c h$-Linie, die den Gegensatz zwischen den hochdeutschen und den niederdeutschen Mundarten markiert.

Auch in der Wortgeographie der deutschen Mundarten herrscht eine Nord/Süd-Gliederung vor. Von den 115 Karten des „Deutschen Wortatlas", die markante Gliederungen aufweisen, tritt nach Hildebrandt (1983)

23 Durrell (1989, S. 89).

24 Durrell (1989, S. 94). 


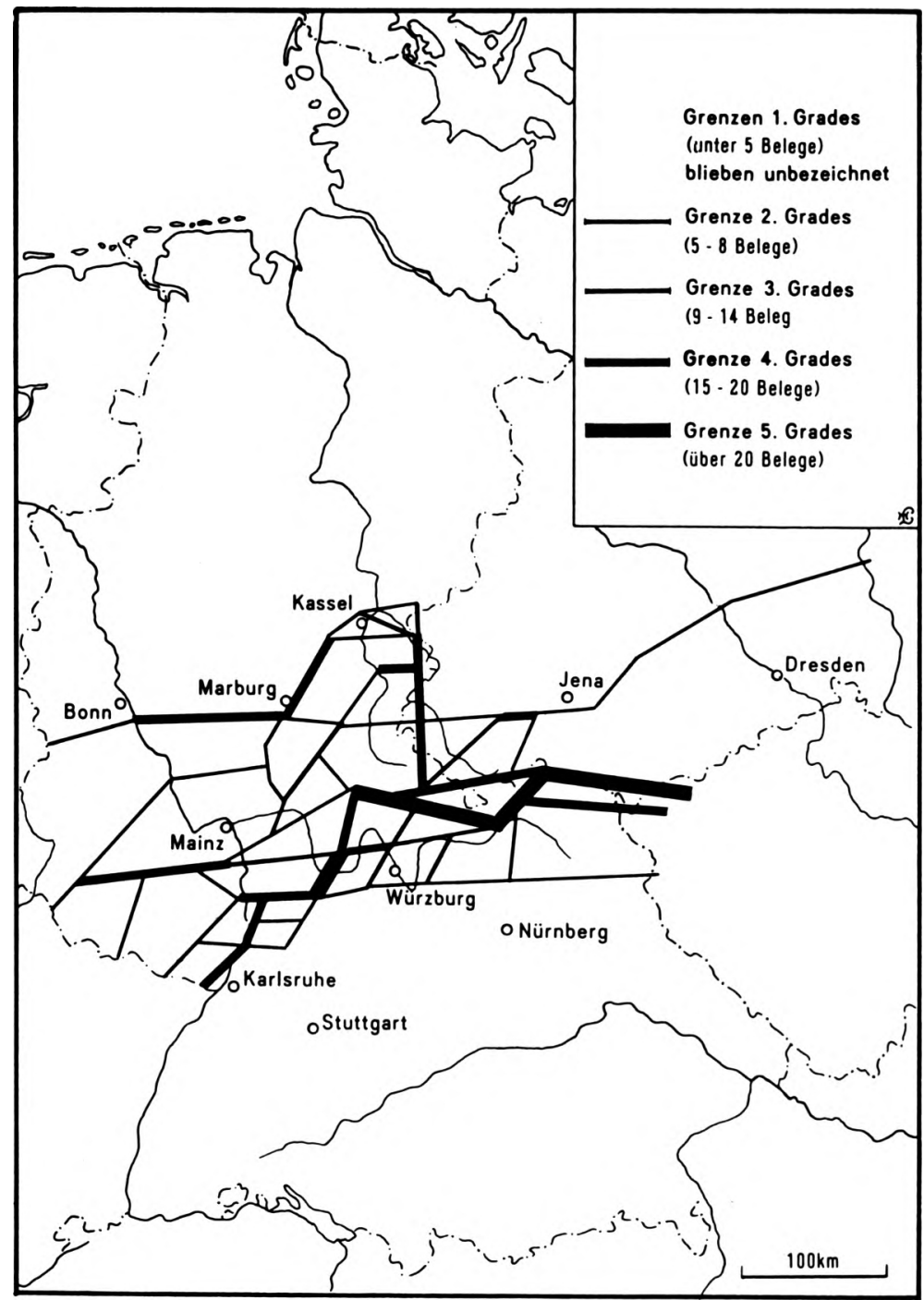

Abb. 2: Kombinationskarte zu Nord/Süd-Gegensätzen in der umgangssprachlichen Lexik auf der Grundlage von 71 Karten des WDU (Bd. 1-2), nach Durrell (1989). 
auf nicht weniger als 77 dieses Muster mehr oder weniger ausgeprägt in Erscheinung. Die Grenzen zwischen den nördlichen und den südlichen Heteronymen verlaufen jedoch wiederum überwiegend viel weiter nördlich, in der Nähe der hochdeutsch/niederdeutschen Dialektgrenze.

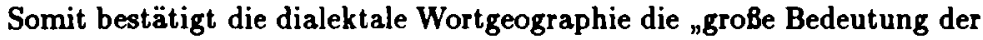
hochdeutsch/niederdeutschen Grenze für die dialektale Strukturierung des deutschen Sprachraums" ${ }^{25}$, sie liefert aber keine Stütze für eine sprachliche Untermauerung der Mainlinie.

Diese sprachliche Untermauerung findet Durrell in der Lexik der Umgangssprachen. Unter den 125 Karten der Bände 1 und 2 des WDU zählt er nicht weniger als 92, die eine Nord/Süd-Gliederung aufweisen; von den 108 Wortkarten sind es $80 .{ }^{26}$ Bei $71^{27}$ der 92 Karten mit Nord/SüdGliederung lassen sich die Geltungsbereiche der Varianten „ohne allzu grobe Vereinfachung" (S. 102) in Form von Isoglossen darstellen. Zu einer vereinfachten Wabenkarte kombiniert, ergeben sie die Karte Abb. 2.

Nach dieser Karte verläuft die bei weitem vorherrschende Scheidelinie zwischen nördlichen und südlichen Formen zunächst entlang der thüringisch-fränkischen Grenze etwas nördlich des Mains, um sich dann in drei Haupt- und mehrere Nebenlinien aufzugliedern. Trotzdem, die Lagerung ist eindeutig: „Wir können [...] zu dem Schluß kommen, daß für die Umgangssprache als heute wichtigste Existenzform der gesprochenen deutschen Sprache eine räumliche Nord/Süd-Gliederung im Bereich der Lexis konstatiert werden kann, die bei aller begrifflichen Ungenauigkeit nicht weit von dem entfernt liegt, was durch die populäre 'Mainlinie' angedeutet wird." 28

Die Karten des dritten und des noch nicht erschienenen vierten Bandes bestärken Durrells Befund. VerhältnismäBig viel weniger von ihnen weisen allerdings die klar umgrenzten Gebiete auf, die in den beiden ersten Bänden vorherrschen. Auf zahlreichen Karten machen ausgedehnte Mischgebiete das Ziehen von Isoglossen unmöglich. Aber immerhin wei-

25 Durrell (1989, S. 97).

26 Durrell (1989, S. 101) nennt die Zahl 81. Jedoch sind in der Auflistung der Karten, auf denen ein nördliches Lexem mehreren sūdlichen gegenübersteht, nur 23 genannt statt der 24, von denen im Text die Rede ist.

2765 Wortkarten und 6 phonologische bzw. morphologische Karten. Die Zahl ergibt sich aus der Zāhlung der von Durrell (1989, S. 102, Anm. 5) identifizierten Karten. Der Text spricht von nur insgesamt 70 Karten.

Durrell (1989, S. 104f.). 


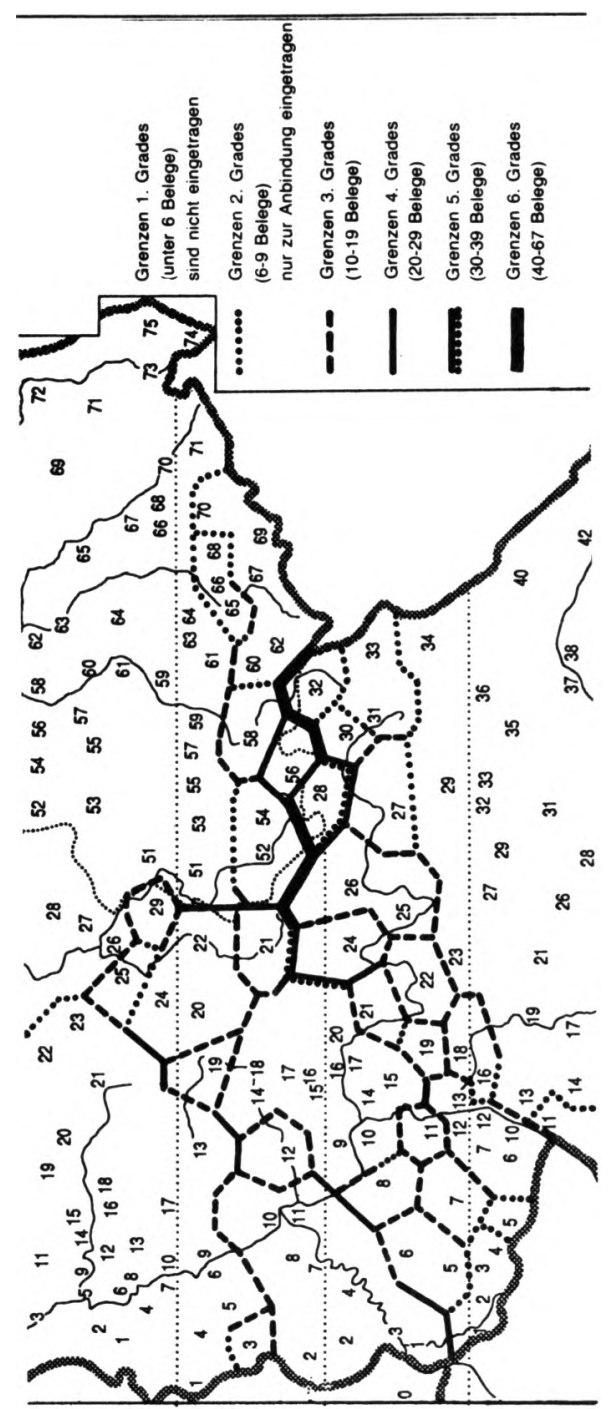

Abb. 3: Kombinationskirte zu Nord/Sūd-Gegeitsätzen in der umgangssprachlichen Lexik auf der Grundlage von 111 Wortkarten des WDU. 
sen von den 119 Wortkarten dieser Bände 42 ein zumindest teilweises nord/südliches Gliederungsmuster mit Übergängen in der Nähe des Mains auf. ${ }^{29}$ Auf der Basis der 65 von Durrell aus den Bänden 1 und 2 herangezogenen Wortkarten plus vier weiterer Karten, die Durrell nicht heranzieht ${ }^{30}$, plus der 42 Karten aus den Bänden 3 und 4 ergibt sich die (vereinfachte) Wabenkarte Abb. 3.

Auf dieser Karte sind Grenzen, die sich aus weniger als sechs Beleggrenzen zusammensetzen, fortgelassen. Grenzen zweiten Grades sind nur eingetragen, um höherrangige Grenzen, die andernfalls ins Leere laufen würden, zu verbinden oder an die Sprach- bzw. Kartengrenze zu führen.

Die um etwa zwei Drittel größere Zahl der Wortkarten ergibt ein Bild, das im wesentlichen mit dem von Durrell eruierten übereinstimmt, jedoch in Details abweicht. Auch auf der präzisierten Karte ist die Scheidelinie in ihrem östlichen Teil am ausgeprägtesten. Auf 58 der 111 herangezogenen Karten verläuft das Isoglossenbündel in dem Bereich der ehemaligen deutsch/deutschen Grenze zwischen Bayern und Thüringen zwischen Hof (F32) und Plauen (E62); zwischen Hof und Saalfeld (E58) sind es 63. Nimmt man die südlich um Hof (2wischen Hof und Marktredwitz, F33) herumschwenkenden Isoglossen hinzu, so erhöht sich die Zahl um weitere 18. Die Linie teilt sich nach Westen zu, wobei in Sonneberg (E56) der Wortgebrauch zum Teil mehr mit dem Süden, in Coburg (F28) zum Teil mehr mit dem Norden übereinstimmt. Das Teilstück westlich der beiden Städte weist dann mit 67 Belegen den größten Kontrast überhaupt auf, um sich vor Fulda (E21) in mehrere Stränge aufzuspalten. Der nach Norden und darauf nach Westen schwenkende Strang schlieft Eschwege (D29) und Kassel (D25), aber nicht HannoverschMünden (D26) und Göttingen (D27) ein. Hier verläuft unsere Grenze also auf eine kurze Strecke im Bereich der hochdeutsch/niederdeutschen

29 Auf einigen (wenigen) Karten gibt es mehr als nur eine in der Nāhe des Mains verlaufende Bezeichnungsgrenze, z.B. bei der Karte 2-93 'der Rotkohl', wo sowohl die Grenze zwischen Rotkohl im Norden und dem südlich anschließenden Rotkraut wie auch die Grenze zwischen Rotkraut und der den ganzen Süden einnehmenden Form Blaukraut heranzuziehen wäre. In manchen Fällen von geographischer Auffächerung ist ebenfalls von zwei in unserem Zusammenhang relevanten Linien auszugehen. Das Kriterium „[einem] Nord- bzw. Süddeutsche[n] [...] 'heimisch' bzw. 'fremd" ' (Durrell 1989, S. 89) ist ohnehin aufzugeben. Den allermeisten Norddeutschen wird die Bezeichnung Rodonkuchen für den Napfkuchen (Karte 2-60) gewiß "fremd" vorkommen (und "tief süddeutsch"). Dennoch muß das Verbreitungsgebiet dieser Bezeichnung dem Norden zugeschlagen werden. "Südlich" ist die Bezeichnung Guglhupf, die im Norden zumindest bekannt ist.

Es handelt sich um die Karten 2-59, 2-66, 2-79, 2-100. 
Dialektgrenze. Marburg (E19) stellt sich im Gegensatz zu Durrells Befund überwiegend zum Süden. Die Grenze im Bereich der Hunsrückschranke ist mit etwa 20 Belegen stärker ausgeprägt als bei Durrell, wohingegen die bei Durrell zwischen Mainz (F10) und Worms (F11) beginnende und nach Westen sich erstreckende Linie auf der präzisierten Karte weniger stark in Erscheinung tritt und sich nach Südwesten zu überhaupt deutlich abschwächt. Die Mehrzahl der vor Fulda nach Süden sich wendenden Beleggrenzen überschreitet den Main nicht südlich von Aschaffenburg (F21), sondern zwischen Gemünden (F24) und Würzburg (F25) und verläuft dann unterhalb von Wertheim (F22) in Richtung Westen. Die Beleggrenze den Rhein hinauf westlich von Bruchsal (G13) und Karlsruhe (G11) ist nicht so stark ausgeprägt und überschreitet den Rhein nicht.

In einem "Schlußbemerkungen" genannten Kapitel seines Beitrags (S. 105-07) deutet Durrell an, daß die politischen und kulturellen Raumbildungen des 19. Jahrhunderts überhaupt erst zu einer auch sprachlichen Ausprägung der „Mainlinie” geführt haben. Gerda Grober-Glück spricht bei ihrer Untersuchung der von Berlin ausgehenden metaphorischen Wendungen in der Umgangssprache von der Mainlinie als geradezu einer "Hemmstelle" 31 , die sich der Ausbreitung von Norden kommender sprachlicher Neuerungen entgegenstellt. Nicht weniger wichtig ist Durrells Beobachtung, daß nördlich dieser Linie umgangssprachlich überwiegend diejenigen lexikalischen Varianten anzutreffen sind, die innerhalb der Standardsprache den höheren Rang einnehmen. ${ }^{32}$ Offenbar habe man es im Bereich der Maingrenze mit einer, jäher abfallenden Stelle entlang der [...] graduellen Abstufung" der umgangssprachlichen Varietäten (siehe Abb.1) zu tun. An dieser Bruchstelle scheidet sich das deutsche Sprachgebiet in zwei Areale, in denen "die gängigste Sprachform [...] durch ihr je anderes Verhältnis zur hochsprachlichen Norm charakterisiert werden kann" (S. 107). Diese in ihrer Bedeutung nicht zu unterschätzende Erkenntnis, von Durrell zunächst noch mit Vorsicht formuliert, wird durch die Karten des dritten und vierten WortatlasBandes bekräftig. Es gilt, sie durch örtliche und kleinregionale Studien beiderseits der "sprachlichen Mainlinie" für größere Bereiche der umgangssprachlichen Grammatik zu verifizieren.

31 Grober-Glück (1975, S. 361).

32 Zu Rangabstufungen innerhalb der Lexik der Standardsprache siehe Eichhoff (1988). 


\section{Nord/Süd-Gliederung in der umgangssprachlichen Phonologie und Morphologie}

Bei den Erhebungen des Belegmaterials und bei der Herstellung der Karten des WDU ging es vor allem um die Darstellung von Worttypen. Lautliche Erscheinungen sind auf den Karten in der Regel nur berücksichtigt, wenn sie gebietsweise verbreitet sind. Ein kursorischer Durchblick erweist rasch, daB solche Erscheinungen praktisch ausschlieblich im Süden des Untersuchungsgebiets auftreten.

Bei den Erhebungen sind jedoch markante Nord/Süd-Unterschiede in der Lautung und Grammatik (die sich in der Regel als südliche Abweichungen von der standardsprachlichen Norm darstellen) auch separat aufgenommen worden. Sie sind zum Teil im Anhang zu Band 2 veröffentlicht. Der Anhang zu Band 4 wird weitere Beispiele bringen. Durrell (1989) hatte sechs der Karten des Anhangs von Band 2 dem Belegmaterial, das der Karte Abb.2 zugrunde liegt, zugeschlagen. ${ }^{33}$ Für unsere präzisierte Karte $\mathbf{A b b} .3$ sind sie nicht berücksichtigt worden. Sie werden stattdessen mit drei weiteren Karten aus dem Anhang von Band 2, einer Karte aus Band 3 und elf Karten aus dem Anhang von Band 4 zu der Karte Abb.4 zusammengefaBt. ${ }^{34}$

Es ist offensichtlich, daf auf dieser Karte die relevanten Linien genau dort Unterschiede in der Phonologie und Morphologie markieren, wo die Karte Abb.3 Unterschiede im Wortgebrauch scheidet. Auch bei den Lauten und Formen ist das Isoglossenbündel im Bereich der alten bayrisch/thüringischen Grenze nördlich des oberen Mains am ausgeprägtesten. Im größten Teil dieses östlichen Drittels bilden sie mit acht bis dreizehn Belegen eine Grenze 4. Grades. Auch hier findet sich die Teilung in einen Strang nördlich um Sonneberg (E56) und einen anderen südlich um Coburg (F28) herum. (Zwischen diesen beiden Städten sind die Unterschiede mit vier Belegen verhältnismäBig gering.) Die Grenze nördlich von Lahn und Mosel ist etwas stärker ausgeprägt als die im Bereich der Hunsrückschranke. Durchschnittlich liegen die Grenzlinien wohl um ein geringes weiter nördlich als bei der Lexik. Sie liegen aber immer noch zentriert dort, wo Durrell aufgrund des lexikalischen Befundes die „sprachliche Mainlinie” angesetzt hat. Ebenso wie bei der Lexik sind bei den Lauten und Formen die nördlichen Formen vorwiegend die, die als standardsprachlich zu gelten haben. Die südlichen gehören einer

33 Durrell (1989, S. 102, Anm. 5).

34 Es handelt sich um die Karten 2-112-13, 2-116, 2-118, 2-120-21, 2-123-25, 3-54, 4-58-59, 4-62, 4-66-67, 4-73-77, 4-79. 


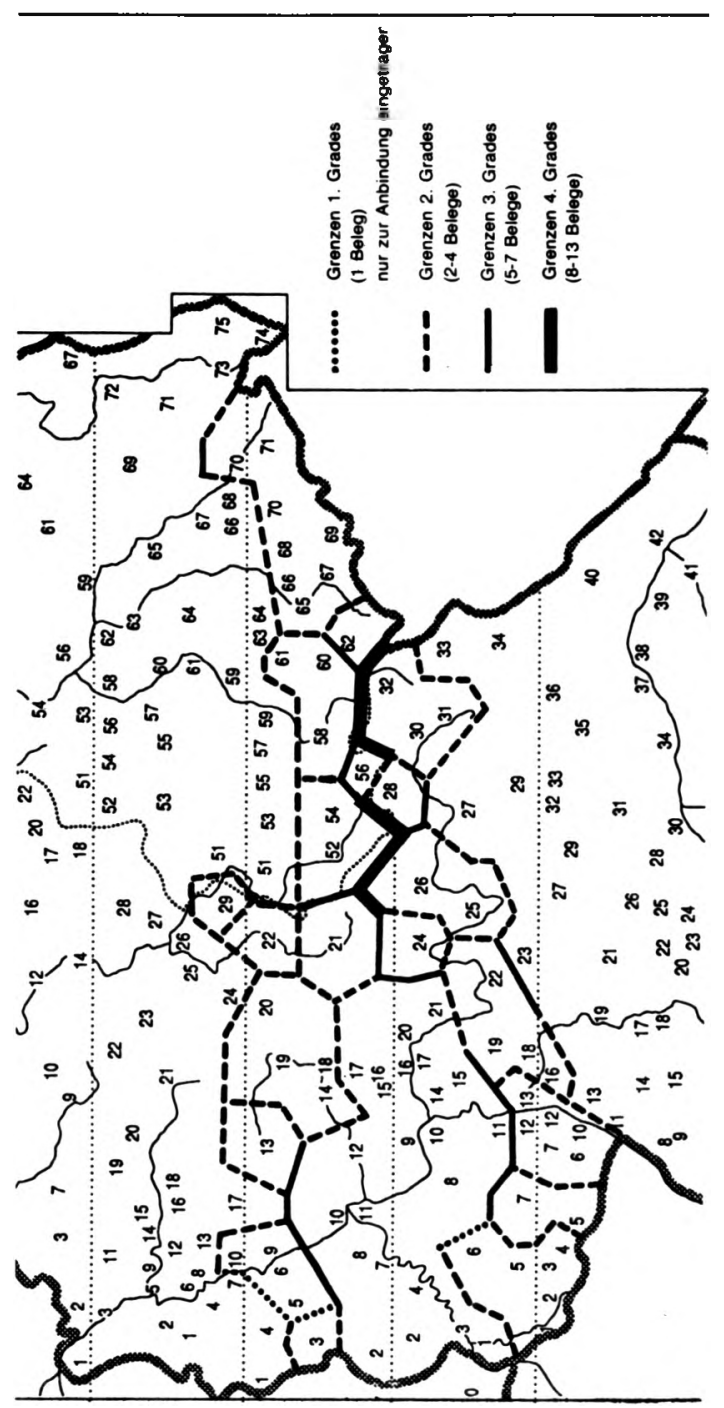

Abb. 4: Kombinationskarte zu Nord/Süd-Gegensaātzen in der umgangssprachlichen Phonologie und Morphologie auf der Grundlage von 21 Karten des WDU. 
höchstens regionalen Norm an, überwiegend aber sind sie als dialektal zu bezeichnen. ${ }^{35}$ Auch in der Phonologie und Morphologie liegt im Bereich des Mains also eine Linie, die ein nördliches, der Standardsprache näherstehendes sprachliches Großareal von einem südlichen Großareal trennt, in dem dialektale Formen vorherrschen. Und auch das phonologische und morphologische Belegmaterial zwingt dazu, auf der Abbildung 1 eine die "Mainlinie" repräsentierende „etwas jäher abfallende Stelle” anzusetzen.

\section{Wortgrenzen und politische Grenzen}

Die für den WDU abgefragten Begriffe konzentrierten sich auf solche, für die auf dem Gebiet der Bundesrepublik und der DDR groBflächig vorkommende landschaftliche Bezeichnungsvarianten zu erwarten waren. Nur für Österreich oder die Schweiz geltende und mit den politischen Grenzen zusammenfallende Besonderheiten des Wortgebrauchs finden ihren Platz sinnvollerweise in Wörterbüchern, nicht in arbeitsaufwendigen Sprachatlanten. Würden diese Besonderheiten dennoch in eine Kombinationskarte eingetragen, so ergäbe sich entlang der deutsch/österreichischen und der deutsch/schweizerischen Grenze ein umfangreiches Linienbündel. Politische Grenzen sind eben auch für Kommunikationsgemeinschaften und damit Sprachräume formativ.

Auf den Karten des WDU zeigt sich Übereinstimmung von politischer Grenze und Wortgrenze bei den Bezeichnungen für das Vormittagsfrühstück am Arbeitsplatz (Karte 1-36), für das im Bairischen Brot$z e i t$ und im deutschen Teil des Alemannischen Vesper gilt. Das Verbreitungsgebiet beider Bezeichnungen endet an der schweizerischen bzw. österreichischen Grenze. Jenseits der Grenze gilt in Österreich Jause, in der Schweiz Znüni. Letzteres kommt auch im ehemals zur Schweiz gehörigen Vorarlberg vor. Jedoch hat die Zugehörigkeit zum österreichischen Staatsgebiet inzwischen schon dazu geführt, daB Jause in drei der vier vorarlbergischen Belegorte ebenfalls als „üblich” bezeichnet wurde. Ein Aussterben von Znüni im Vorarlberg und damit ein Zusammenfall von Wortgrenze und Staatsgrenze ist abzusehen. Ein solcher Zusammenfall findet sich wiederum bei der Bezeichnung Halbmittag, das in Südtirol, und nur hier, verwendet wird.

35 Zum Beispiel das Diminutivsuffix, Karte 3-54. Die nördlichen Formen ein bißchen, ein bißje sind standardsprachlich oder stehen der Standardsprache nahe. Die südlichen Formen, ein bissl, ein bissle sind deutlich dialektal. Standardsprachliches -lein kommt in den Umgangssprachen bei diesem Wort nicht vor, übrigens auch nicht als Diminutivsuffix bei Haus, vgl. Karte 2-121. 


\subsection{Die deutsch/österreichische Grenze als Wortgrenze}

Obwohl die Feststellung von Wortgrenzen entlang der deutsch/österreichischen Grenze nicht Ziel der Abfragungen für den WDU war, tritt diese Grenze auf einer Vielzahl der Karten zumindest in Teilbereichen in Erscheinung. Die politische Grenze ist Grenze zwischen den Bezeichnungen (typisiert) sich erkälten/sich verkälten auf dem Gebiet der Bundesrepublik und dem österreichischen Heteronym sich verkühlen (Karte 1-6), zwischen Bulldog und Traktor (Karte 1-12), Schreiner und Tischler (Karte 1-20), Kamin und Rauchfang, (Karte 1-23, die westlichen Bereiche Österreichs ausgeschlossen; ähnlich Kaminkehrer und Rauchfangkehrer, Karte 1-22), Gang und Vorraum, Vorzimmer (Karte 1-25, die westlichen Bereiche ausgeschlossen), Pinte, Beize und Beisel (Karte 1-32), Schusser und Kugel, Kugerl (Karte 1-50), Seil, Springseil und Springschnur (Karte 1-51), Negerkuß, Mohrenkopf und Schwedenbombe (Karte 2-62), Bonbon, Guatl und Zuckerle (Karte 2-63), Fleischküchle, Fleischpflanzl und Fleischlaberl (Karte 2-65), Putzlumpen, Hadern und Fetzen (Karte 2-80), Schnake, Staunze und Gelse (Karte 2-101), Mäuserl und das närrische Bein, das damische Aderle (Karte 3-6 'der Musikantenknochen'), Geldbeutel und Geldtasche (Karte 3-15), Häuslehupfen, Kastlhupfen und Tempelhupfen (Karte 3-27 'Himmel und Hölle'), Karussell und Ringelspiel (Karte 3-29, die westlichen Bereiche ausgeschlossen), Federmäppchen und Federpennal (Karte 3-32), sich schicken und sich tummeln, sich schleinen (Karte 3-42 'sich beeilen'), Hausschuhe, Pantoffeln und Patschen, Schlapfen (Karte 4-8), Mülltonne und Müllkübel, Mistkübel (Karte 4-17), Hörndl und Kipferl, Kipfl (Karte 4-21), Pfann(en)kuchen und Omelett, Palatschinke (Karte 4-24), Hackfleisch und Faschiertes (Karte 4-34), Bierfilzl und Biertatz(er)l(Karte 4-44; Bierdeckel streut), Blumenkohl und Karfiol (Karte 4-49), Rosenkohl und Sprossenkohl, Kohlsprossen (Karte 4-50), Aprikose und Marille (Karte 4-53) - um nur die deutlichsten Beispiele zu nennen.

Diese umgangssprachlichen Wortgrenzen sind allerdings zum Teil das Ergebnis eines erst auf dieser sprachlichen Ebene ganz durchgeführten komplementären territorialen Ausgleichs. Damit ist ein ProzeB gemeint, bei dem Bezeichnungen, die innerhalb einer größeren Kommunikationsgemeinschaft nur von einer Minderheit verwendet werden, in der Regel weiter zurückgehen und schließlich aussterben. Auf basisdialektaler Ebene reicht nach Scheuringer (1995) das Gebiet zum Beispiel von Schreiner über die politische Grenze hinweg nach Oberösterreich hinein, während umgekehrt das in den Umgangssprachen (und Dialekten) Österreichs herrschende Heteronym Tischler im äußersten Osten Niederbayerns basisdialektal in einem zehn bis zwanzig Kilometer breiten Streifen auf die 
deutsche Seite hinüberreicht. Hier spiegeln sich historische Grenzverschiebungen, die im Laufe der Zeit auf den höheren sprachlichen Ebenen eine Art ${ }_{n}$ sprachliche[r] Bereinigung der Staatsgrenze" (Scheuringer) zur Folge hatten.

\subsection{Die deutsch/schweizerische Grenze als Wortgrenze}

Auch die deutsch/schweizerische Grenze markiert in vielen Fällen Bezeichnungsunterschiede. Völlige Übereinstimmung herrscht dabei nicht nur bei Bezeichnungen, die durch administrative Verfügung gestützt sind (schweiz. Führerausweis, dt. Führerschein, Karte 3-48), sondern auch bei Begriffen jungen Ursprungs wie schweiz. Poulet, dt. Brathährchen (Karte 4-34; die aus dem Dialekt stammende Bezeichnung Güggeli streut beiderseits der Grenze), und Panaschee, dt. Radler (Karte 4-40 'Mischgetränk aus Bier und Limonade'). Die meisten mit der politischen Grenze übereinstimmenden umgangssprachlichen Bezeichnungsgrenzen betreffen jedoch durchaus auch älteres Wortgut: Estrich gegenüber Bühne, Speicher (Karte 1-24 'der Dachboden'), viertel 6 gegenüber dreiviertel 6 (Karte 1-39), Schläckstängel gegenüber Lutscher (Karte 2-64), Bitzgi gegenüber (Apfel-)Butzen (Karte 2-97), Narrebeinli gegenüber Musikantenknochen, Mäusle (Karte 3-6), Portemonnaie gegenüber Geldbeutel (Karte 3-15; letztere Bezeichnung gilt nur in Süddeutschland), Velofahrer gegenüber Radfahrer (Karte 3-17), Nuggi gegenüger Schnuller (Karte 323), Etui gegenüber Mápple (Karte 3-32 'das Federmäppchen'), krampfen gegenüber schuflen (Karte 3-49), Garette gegenüber Schubkarren (Karte 3-50), Ni(i)del, Niidle gegenüber Rahm (Karte 4-28; Rahm streut auch in der Schweiz). Es kann an dieser Stelle nicht untersucht werden, wie alt dieser Zusammenfall von Wortgrenze und Staatsgrenze in jedem Fall ist. Möglicherweise handelt es sich auch hier um das Übergreifen der (in der jeweiligen Kommunikationsgemeinschaft) mehrheitlich verwendeten $\mathrm{Be}$ zeichnung auf das Gebiet der minderheitlich verwendeten Bezeichnung im Zuge des komplementären territorialen Ausgleichs. Es fallt jedoch auf, daß in nicht wenigen Fällen als schweizerisch zu bezeichnende Besonderheiten in das südliche Baden-Württemberg hineinreichen, besonders die Städte Lörrach und Waldshut einschließend: Maitli gegenüber Mädle (Karte 1-2), lu(e)g! gegenüber guck! (Karte 1-8), wischen gegenüber kehren (Karte 1-16 'fegen'), Znüni gegenüber Vesper (Karte 1-35 'das Vormittagsfrühstück'), grüezi gegenüber grüß Gott (Karte 1-47 'Gruß beim Betreten eines Geschäfts'), Weggli gegenüber Weck(en) (Karte 259), Härdöpfelstock gegenüber Kartoffelbrei (Karte 2-67), Zündhölzli gegenüber Streichholz (Karte 2-75), Zapfe gegenüber Korken (Karte 2-76), Schulsack, Schultheek gegenüber Schulranzen (Karte 2-88), pressieren gegenüber sich beeilen (Karte 3-42), Finken gegenüber Schlappen, Haus- 
schuhe (Karte 4-8), Nastuch gegenüber Taschentuch (Karte 4-9), Omelett(e) gegenüber Pfannkuchen (Karte 4-24) undGschwellti gegenüber Pellkartoffeln (Karte 4-46). In den dialektnahen Umgangssprachen dieser Gebiete wirkt sich die Grenze zwischen den hochalemannischen und den niederalemannischen Dialekten aus, die auch kulturell eine Rolle spielt und etwa dreißig Kilometer nördlich der politischen Grenze verläuft. Es ist jedoch abzusehen, daB auch hier Wortgrenzen und nationale Grenzen einander angleichen werden. Dies vor allem aufgrund des Umstandes, $\mathrm{da} B$ in Deutschland die Umgangssprachen sich zunehmend der Standardsprache nähern, während die Schweiz in der alltäglichen Kommunikation bei der Verwendung des Dialekts beharrt.

\subsection{Die deutsch/deutsche Grenze von 1945-1989 als Wortgrenze}

Die Aufnahmen für den WDU waren abgeschlossen, bevor im Jahre 1990 die formelle Wiedervereinigung der beiden Teile Deutschlands vollzogen wurde. Wenn, wie das Beispiel der deutsch/österreichischen und der deutsch/schweizerischen Grenze zeigt, die politische Grenze die Bildung sprachlicher Grenzen zur Folge hat, wird man fragen wollen, in welchem Umfang sich auch die deutsch/deutsche Grenze sprachlich schon ausgewirkt hatte.

Bekanntlich ist der Streit um die „sprachliche Teilung” auf beiden Seiten mit wechselnden, zumeist ideologisch gefärbten Argumenten geführt worden. ${ }^{36}$ Es ging dabei vor allem um Neuwörter, aber auch um Bedeutungsentwicklungen von Wörtern, die die Zugehörigkeit der beiden deutschen Staaten zu unterschiedlichen politischen Systemen widerspiegelten. Der neutrale Wortschatz war nur wenig betroffen. Aber auch hier stand die sprachliche Entwicklung nicht still. Mit der Einführung neuer Sachen kamen neue Bezeichnungen. Gebrauchsgegenstände aus Kunststoff zum Beispiel: In der Bundesrepublik bürgerte sich dafür nach und nach die aus englisch plastics entlehnte Bezeichnung das Plastik ein, in der DDR der Plast, daraus später die in die Funktion des Singulars übergetretene Pluralform die Plaste. ${ }^{37}$ Neu waren auch die Bluejeans, für die im Norden der Bundesrepublik zunächst Nietenhose, in der DDR Niethose gebräuchlich wurde, bevor es dann überall nur noch Jeans hieB. Die Kombine und der Mähdrescher sowie das Brathähnchen und der Broiler kommen noch hinzu.

36 Im Überblick zuletzt noch, mit Bibliographie, Russ (1994, S. 100-120).

37 Dazu ausführlicher Eichhoff (1980, S. 163-66). 
Größer war die Zahl der Begriffe, bei denen die Grenzen unterschiedlichen Wortgebrauchs nicht, genauer gesagt: noch nicht mit der Grenze zwischen den beiden Staaten übereinstimmten. Hätte die Grenze noch zwei oder drei Generationen länger Bestand gehabt, so wäre es gewib auch hier zu weitgehendem komplementärem territorialem Ausgleich gekommen. Die Abbildung 5 vereinigt die 14 vom WDU erfaBten

Beispiele zu einer Kombinationskarte. ${ }^{38}$ Im Süden, von der deutsch/ tschechischen Grenze bis auf die Höhe von Göttingen (D27), fallen auf dieser Karte die Wortgrenzen überwiegend mit der politischen Grenze zusammen. Dann teilt sich das Isoglossenbündel in einen östlichen und einen westlichen Strang. Der östliche Strang ( 7 Belege) läuft weiterhin entlang der Staatsgrenze, der westliche (4 Belege) führt jedoch an dem Industriegebiet zwischen Goslar (C18) und Wolfsburg (C21) westlich vorbei. Die Elbe zwischen Wittenberge (B55) und Lauenburg (B23) bildet mit drei Belegen ${ }^{39}$ eine nur schwache Grenze. Mehrere Beleggrenzen verlaufen hier weiter östlich innerhalb des Gebietes der ehemaligen DDR und signalisieren damit noch die enge Bindung dieses Landstriches an den westlich anschließenden Hamburger Raum. Erst östlich von Lübeck vereinigen sich die Stränge wieder. Man darf gespannt darauf sein, wie sich die Sprachverhältnisse in diesem Gebiet nach der Wiedervereinigung entwickeln. Manche Bezeichnungen, darunter der Broiler, werden verschwinden. Alster(wasser) und Radler greifen nach vorläufigen Beobachtungen rasch auf die jeweils angrenzenden Gebiete der neuen Bundesländer über. Eine umfasserendere Antwort muB späteren Untersuchungen vorbehalten bleiben.

38 In dieser Karte sind die Beispiele Nietenhose/Niethose (Karte 2-86) und Führerschein/Fahrerlaubnis (Karte 3-48) nicht enthalten, weil die Gegensätze schon vor der Wiedervereinigung nicht mehr vorhanden waren. Dazu Eichhoff (1978, S. 25), und Eichhoff (1993, S. 32). - Protze (1990, S. 142) sieht komplementären territorialen Ausgleich auch bei Senf gegenüber Mostert als möglich an. Seine Karte (S. 141) stützt diese Ansicht. Sie beruht jedoch auf Aufnahmen auch aus kleineren Orten, in denen der alltägliche Sprachgebrauch möglicherweise stärker bei konservativen Formen beharrt. Unsere Karte (2-71), die das sprachliche Verhalten in den größeren und gewiß trendsetzenden Städten wiedergibt, dokumentiert ein deutliches Ūberwiegen von Senf auch auf dem Gebiet der DDR.

39 Es handelt sich um die Grenzen auf den Karten 2-77 'das Plastik', 4-20 'die belegte Brotscheibe', und 4-34 'das Brathähnchen'. 


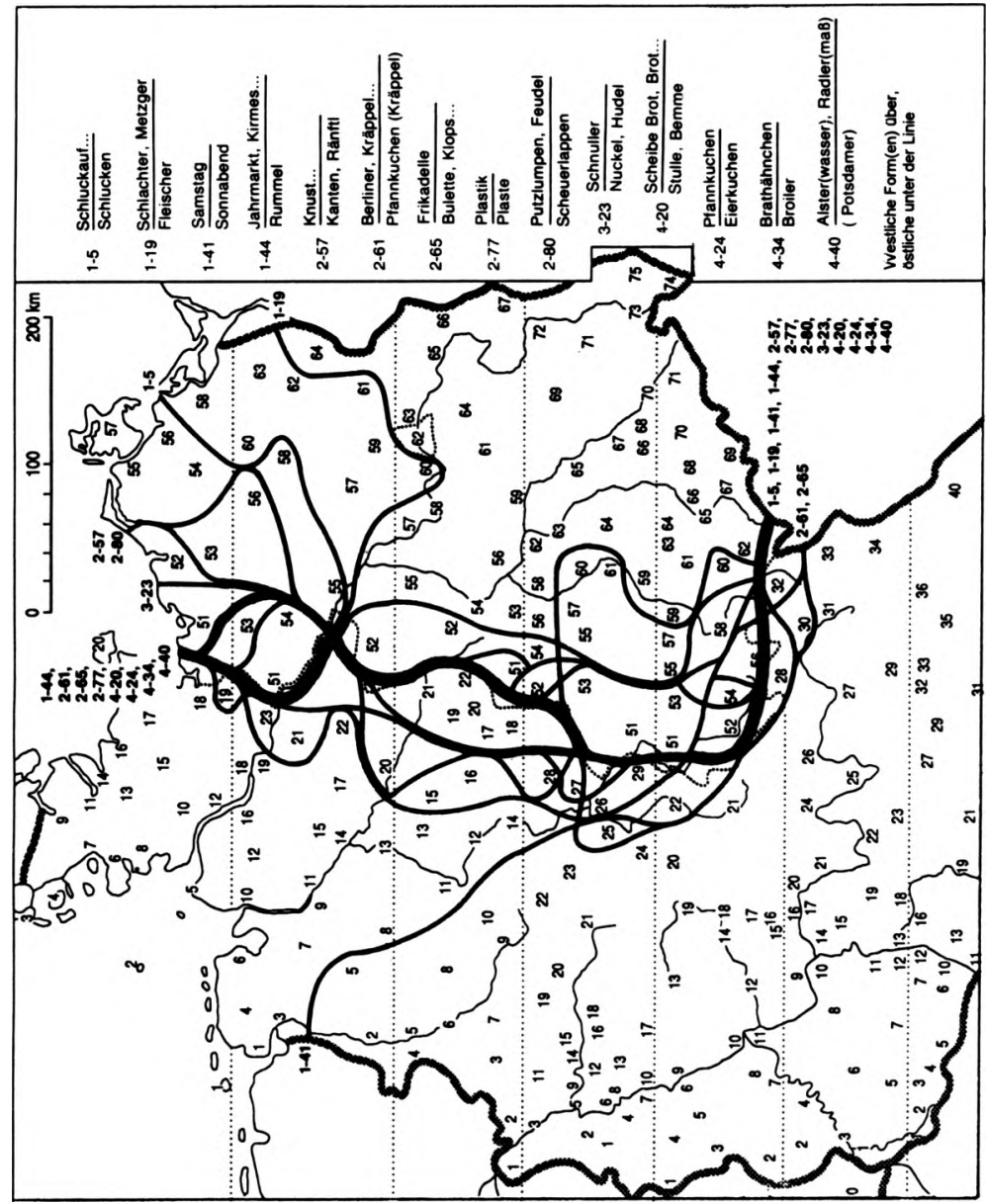

Abb. 5: Kombinationskarte zu Ost/West-Gegensātzen i der umgangssprachlichen Lexik im Bereich der deutsch/deutschen Grenze 1945-1989, auf der Grundlage von 14 Karten des WDU. 


\section{Lexikalische Varianten der Umgangssprachen in ihrem Verhältnis zur dialektalen Lexik}

Das für den WDU systematisch über das gesamte deutsche Sprachgebiet erhobene Material erlaubt es nunmehr erstmals, aus der vergleichenden Untersuchung umgangssprachlicher und dialektaler Wortareale Schlüsse hinsichtlich sprachlicher Entwicklungen zu ziehen. Allerdings müssen im Norden bzw. Süden die unterschiedlichen Voraussetzungen zu unterschiedlichen Problemstellungen führen. Nur im Norden, im Bereich der niederdeutschen und zum Teil noch mitteldeutschen Dialekte, besteht zwischen diesen und den (hochdeutschen) regionalen Umgangssprachen der qualitative Unterschied, der Sprachentwicklungen großräumig sichtbar machen kann. Im Süden schafft der WDU eher neue Voraussetzungen für die Untersuchung von Stadtsprachen gegenüber Landmundarten, noch weiter südlich, in der Schweiz, lassen sich mit Hilfe der WDU-Karten diachronische Vergleiche mit früheren Dialektaufnahmen durchführen.

6.1 Zur Herkunft lexikalischer Varianten in den Umgangssprachen Norddeutschlands

Jan Goossens (1979) hat, von den Karten des WDU ausgehend, die Frage nach der Herkunft (endogen oder exogen) des umgangssprachlichen Wortschatzes im Geltungsbereich des Niederdeutschen gestellt. Aufgrund von 24 Begriffen, die sowohl im WDU (Band 1 und 2) wie auch im "Deutschen Wortatlas" (DWA) behandelt sind und deshalb den Vergleich ermöglichen, trifft er die Feststellung, daB erstens, immer „wenn (fast) der gesamte niederdeutsche Mundartraum nur über einen einzigen Ausdruck verfügt, [...] dieser in verhochdeutschter Gestalt auch Bestandteil der norddeutschen Umgangssprache geworden" ist (S. 46). Beispiele sind die Bezeichnungen Junge, kneifen, fegen, Klempner, Streichholz, Meerrettich, Rotkohl, Ziege und Mücke.

Ohne daB der Nachweis an dieser Stelle im einzelnen geführt werden kann, dürften aus den Bänden 3 und 4 die folgenden drei Bezeichnungen in dieselbe Kategorie fallen:

Allag (zu erschlieBen aus der Karte 3-40 'am Wochentag/wochentags'). Die Bezeichnung Alltag gilt in den Umgangssprachen bis an die Westgrenze und die Grenze zu Dänemark, wo in den Mundarten Werkeltag und ähnliche Bezeichnungen gelten.

auswringen (Karte 4-15). Dieses Wort, in seinem ersten Teil verhochdeutscht aus niederdeutsch utwringen, nimmt trotz Bewahrung des ty- 
pisch niederdeutschen $w r_{-40}$ heute fast die gesamte nördliche Hälfte des deutschen Sprachgebiets ein. Die mundartlich im Süden des westlichen Niederdeutschen gültige Form utdrücken ist in den Umgangssprachen nicht mehr vertreten. Schon auf der DWA-Karte reicht das Wort in starker mundartlicher Aufsplitterung und z.T. in Konkurrenz mit anderen Formen bis an die Mosel, wo sich südlich ausdrehen anschlieBt. Die Mosel ist auch in den heutigen Umgangssprachen die Scheide zwischen ausdrehen im Süden und dem jetzt einheitlich als auswringen erscheinenden nördlichen Heteronym. Wir haben hier einen der seltenen Fälle, wo ein Wort mit erkennbar niederdeutscher Lautung trotz vorhandener "hochdeutscher" landschaftlicher Synonyme (ausdrehen, auswinden und ausringen) standardsprachlich den höchsten Rang einnimmt.

Goossens' Regel ist nicht ohne Ausnahme. Das niederdeutsche Wort für den Topf, Pott, ist trotz seiner Verbreitung über praktisch das gesamte niederdeutsche Sprachgebiet hinweg (mit Ausnahme eines von Süden über Berlin hinausreichenden Keils) nicht in die norddeutschen Umgangssprachen aufgenommen worden. Obwohl der WDU (Karte 4-12) den Begriff als Bezeichnung für ein GefäB für Milch, der DWA dagegen als 'irdener Topf' abgefragt hat, sind die Karten vergleichbar. Der Grund für die Zurückweisung des niederdeutschen Wortes dürfte in der lautlich „unmöglichen” Form zu suchen sein, die die Übertragung ins Hochdeutsche zur Folge hätte. Stattdessen ist im gesamten niederdeutschen Raum, soweit nicht (möglicherweise sachlich unterschiedene) Bezeichnungen wie Kanne und Krug auftreten, umgangssprachlich die aus dem Ostmitteldeutschen stammende, heute standardsprachliche Form Topf vertreten.

Als zweites untersucht Goossens Fälle, in denen der niederdeutsche Mundartraum zwei groBräumige Heteronyme aufweist. In diesen Fällen ist zu beobachten, daß Dialektwörter, die nur im westlichen Teil des Gebiets vorkommen, "nicht den Status der Umgangssprache erreichen oder [...] es schwer [haben], diesen Status zu behalten" (S. 49). Sie werden durch Bezeichnungen aus den östlichen und südöstlichen Bereichen zurückgedrängt. Beispiele für expandierende Bezeichnungen sind pfeifen, heute morgen, Samstag und bedingt auch Tischler.

Aus den Bänden 3 und 4 treten hinzu:

Peitsche (Karte 3-51). Die westniederdeutsche Bezeichnung $S$ (ch)weep u.ä. galt in den meisten Mundarten zur Zeit der Aufnahmen für den DWA nur noch neben Pietsch, der aus dem Sorbischen zunächst ins Ost-

40 Die schwierige Lautverbindung wird, auch in den Dialekten, von den Sprechern z.T. durch fr- ersetzt. 
mitteldeutsche übernommenen Bezeichnung. In den Umgangssprachen ist das Wort vollkommen verschwunden.

Pilz (Karte 4-48). Die in den westniederdeutschen Mundarten verbreitete, aber auch die nördlichen Dialekte des ostniederdeutschen Raums einnehmende Bezeichnung Poggenstaul ist als umgangssprachliche Form nicht mehr gemeldet worden. Der ausgefallene Charakter der Metapher ( "Krötenstuhl”) dürfte seiner Aufnahme ins Hochdeutsche Schwierigkeiten entgegengesetzt haben. Das Ersatzwort hat wiederum im Ostmitteldeutschen seinen Ursprung; die Allgemeinbezeichnung im Süden ist bekanntlich Schwamm.

AbschlieBend diskutiert Goossens fünf Fälle, in denen die niederdeutsche Dialektlandschaft in mehrere Wortgebiete aufgeteilt ist. Die entsprechenden Karten des WDU sind 'das Mädchen' (1-2), 'die Schaufel' (1-14), 'die Rinde der Brotscheibe' (2-56), 'der Schnürsenkel' (2-87) und 'die Möhre' (2-89). Mit Ausnahme der Karte 'Mädchen' weisen hier auch die Umgangssprachen in Norddeutschland mehrere Gebiete auf, z.B. Schippe bzw. Schüppe im westlichen und östlichen Bereich neben Schaufel im zentralen norddeutschen Raum auf Karte 1-14. Diesen Fällen entsprechen aus Band 3 die Karte 'der Schnuller' und aus Band 4 die Karte 'die Sahne'.

Karte 3-23 'der Schnuller'. In den niederdeutschen Mundarten herrscht die Bezeichnung Lutscher in einem breiten nördlichen Streifen vor, während im südwestlichen Niederdeutschen Sauger und im südöstlichen Nuckel gelten. Letztere Bezeichnung reicht aus dem südlich angrenzenden Ostmitteldeutschen herein, in dessen Südteil sich wiederum mehrere kleinräumige Bezeichnungen, vor allem Huttel, finden. Nördlich des Nuckel-Gebiets zur Ostseeküste hin streut Titt stark ein. Zwischen den Erhebungen für den DWA und denen für den WDU ist an die Stelle des gefüllten Stoffbeutelchens, das dem Kind zum Saugen gegeben wurde, der Gummisauger getreten. Damit ist auch die Bezeichnungslandschaft grundlegend bereinigt worden. Im gesamten westniederdeutschen Raum, aber hinab bis an die Schweizer Grenze gilt jetzt in den Umgangssprachen fast ganz einheitlich Schnuller. Die einzige konkurrierende Bezeichnung ist Nuckel, die nicht nur den Süden des ostmitteldeutschen Gebiets erobert hat, sondern auch den gesamten ostniederdeutschen Raum bis an die Ostseeküste. Es war geradezu ein Kennwort der DDR geworden. Die Bezeichnung Schnuller ist in den Dialekten im Schwäbischen, Ostfränkischen und Nordbairischen, aber immer in Konkurrenz mit anderen Bezeichnungen, verbreitet. In den hochdeutschen Umgangssprachen muB sie rasch den Rhein hinauf und an der Westgrenze entlang vorgestoBen sein, unterstützt möglicherweise durch den kommerziellen Vertrieb des 
nun industriell hergestellten Produkts. Offenbar wurde eine Bezeichnung gebraucht, die sich von der des alten Saugers mit hinreichender Deutlichkeit unterschied.

Karte 4-28 'die Sahne'. Der größte Teil des niederdeutschen Mundartraums teilt mit dem Süden die Bezeichnung Rahm. Sie kommt auch in den norddeutschen Umgangssprachen vor, ist jedoch insgesamt seltener als die aus dem Ostmitteldeutschen vorstoßende Bezeichnung Sahne. Überraschend ist das Beharrungsvermögen der kleinräumig verbreiteten mundartlichen Bezeichnungen Schmant und Flott. Beide sind auch in den Umgangssprachen vertreten, wenngleich letzteres nur viermal um Braunschweig gebucht wurde. Schmant findet sich umgangsprachlich im Westfälischen, Moselfränkischen und im Nordhessischen, z.T. in kompakten Gebieten. Möglicherweise macht sich der Umstand geltend, daB in der Auseinandersetzung zwischen dem hier leicht mundartlich klingenden Rahm und dem noch nicht akzeptierten Sahne die Bezeichnung Schmant als Ausweichform sich behaupten kann.

Insgesamt bestätigt die Mehrzahl der Beispiele aus den Bänden 3 und 4 Goossens' Folgerung, daß die Umgangssprachen in Norddeutschland

„ihr Wortgut meistens aus den niederdeutschen Mundarten übernommen [haben]. Je großräumiger der Dialektausdruck, um so besser seine Chancen in der Umgangssprache. Ostniederdeutsche Wörter setzen sich leichter durch als westniederdeutsche [...]. Von den angrenzenden mitteldeutschen Bereichen hat $\mathrm{zw}$ weifellos das Obersächsische den stärksten EinfluB auf die Herausbildung des norddeutschen umgangssprachlichen Wortschatzes gehabt." (S. 50f.)

6.2 Varianten der umgangssprachlichen Lexik in ihrem Verhältnis zum Dialekt im mittleren und südlichen Teil des Sprachgebiets

Zum Verhältnis zwischen den Umgangssprachen und Dialekten im mittel- und süddeutschen Raum liegen noch keine die Wortatlaskarten vergleichend auswertenden Arbeiten vor. Im folgenden seien einige Beobachtungen formuliert, die Ausgangspunkt weiterreichender Untersuchungen sein könnten.

Im mitteldeutschen Raum verwischt sich die qualitative Trennung zwischen den Dialekten und den Umgangssprachen, die die sprachlichen Verhältnisse im Norden bestimmt. Wie die Abbildung 1 illustriert, stehen hier die Umgangssprachen den Dialekten näher, Übergänge zwischen beiden erfolgen leichter. So finden sich in den Umgangssprachen des mitteldeutschen Raumes Formen mit unverschobenem $p$ oder $t$, die im Norden gemieden bzw. als dialektal sanktioniert würden. Ein Beispiel ist die Bezeichnung kneipen u.ä. mit unverschobenem p, die auf der Karte 1-10 
'kneifen' vom Obersächsischen über das Thüringische bis ins nördliche Hessen hinein streut. Daß es sich hier nicht um mundartliche Irrläufer, sondern um legitime, den Umgangssprachen zuzurechnende Formen handelt, wird durch die Aufnahme von kneipen als Stichwort in die DudenBände ${ }^{41}$ sozusagen sprachamtlich bestätigt.

Auf derselben Karte zieht sich von Basel das Rheintal hinauf die Variante pfetzen. Sie geht in einer Linie westlich von Karlsruhe, Heidelberg und Michelstadt dann in die Form petzen über - übrigens der einzige Fall, wo auf den Karten des WDU eine Lautverschiebungsgrenze wenigstens teilweise sichtbar wird. Auch hier hat sich die Duden-Redaktion entschlossen, das umgangssprachliche Wort mit mundartlicher Lautung (mit der Verbreitungsangabe "westmitteldeutsch") in die Wörterbücher aufzunehmen. ${ }^{42}$

Unverschobenes $t$ findet sich in der Bezeichnung Schnute für den AusguB der Kaffeekanne (Karte 2-78), die am unteren Rhein recht kompakt vorkommt. Die Wörterbücher kennen das Wort nur in den Bedeutungen 'Mund, besonders eines Kindes' und 'Gesichtsausdruck, der Verdrossenheit, Enttäuschung, Beleidigtsein o.ä. ausdrückt'. ${ }^{43}$ Die Form mit Standardlautung, Schnauze, ist im Obersächsischen verbreitet, streut aber auch über die Mainlinie hinweg in den süddeutschen Raum.

In der Schweiz ist auch in den Städten die Mundart Sprache des täglichen Umgangs. Die Karten des WDU können hier allenfalls dazu dienen, zum Vergleich mit den nunmehr fast ein halbes Jahrhundert zurückliegenden Aufnahmen für den "Sprachatlas der deutschen Schweiz" herangezogen zu werden. Ein Beispiel: Auf der Karte 'kneifen' verzeichnet der WDU für die östliche Schweiz chlübe, für den westlichen Teil der deutschsprachigen Schweiz chlemme, im Zentrum chlimse und chnüüble, für das Wallis zwengge. Genau diese Bezeichnungen finden sich in eben den Gebieten auf der entsprechenden Karte des "Sprachatlas der deutschen Schweiz".44 Sogar die "fremde" Form petzen in Basel wird dort

41 Z.B. Duden Deutsches Universalwörterbuch (1989, s.v).

42 Duden Deutsches Universalwörterbuch (1989, s.v). - Auch das weit weniger verbreitete pfetzen ist vertreten, mit der Verbreitungsangabe „landschaftlich”. Möglicherweise brauchte man das Wort, um das verbreitetere petzen als seine „Nebenform” kennzeichnen zu können. Die mittelfränkische Variante pitschen ist der Aufnahme nicht für würdig befunden worden.

43 Duden Deutsches Universalwörterbuch (1989, s.v).

44 Hotzenköcherle (1962-, Bd. IV, Karte 92). 
bestätigt. Ob die Übereinstimmung stets so vollkommen ist und wo im einzelnen Verschiebungen stattgefunden haben, werden Studien an umfangreicherem Material zeigen müssen.

\section{Norddeutsche umgangssprachliche Lexik in ihrem Verhältnis zur Standardsprache}

Bei der Vorstellung des umgangssprachlichen Stufenmodells (Abb.1) im ersten Band des WDU wurde darauf hingewiesen, daB die für die Laute und Formen gültige Vorstellung: „Im Norden der Standardsprache näher, im Süden ferner" nicht unbedingt auch für den Wortgebrauch zutrifft. ${ }^{45}$ Auch der Norden kennt regional begrenzte, zum Teil (aber nicht in jedem Fall) aus den niederdeutschen Dialekten stammende Bezeichnungen wie Trecker (Karte 1-12 'der Traktor'), Mostrich (Karte 2-71'der Senf'), Wurzel (Karte 2-89 'die Möhre'), Bickbeere (Karte 2-96 'die Heidelbeere'), klönen (Karte 3-9 'sich unterhalten'), die dem Standarddeutschen nicht oder nur unter Vorbehalten wie "landschaftlich” angehören und von Sprechern mit ausgeprägtem Sprachempfinden in formellen Situationen oder bei der Kommunikation mit Personen aus anderen Teilen des deutschen Sprachgebiets gemieden werden.

Gunter Müller (1980) hat in einer komputergestützten Untersuchung die Frage zu beantworten versucht, ob die Distanz zwischen lexikalischem Standard und umgangssprachlicher Lexik im norddeutschen Sprachraum regional differenziert ist, d.h., ob es Gegenden gibt, in denen die lexikalische Komponente der Umgangssprache mehr standardsprachlich akzeptiertes Wortgut enthält als in anderen. Zu diesem Zweck wählte er 67 für eine derartige Untersuchung geeignete Karten aus den beiden ersten Bänden des WDU aus und errechnete für jeden der 201 Orte in den Zonen A bis E der WDU-Grundkarte einen Faktor, der sich aus der Klassifikation der gemeldeten Formen als "regional”, „umgangssprachlich" usw. oder auch deren Fehlen in dem Wörterbuch von Wahrig ergibt. Durch Schwarzfärbung eines dem Faktor entsprechenden Kreissektors und dessen Eintragung in die WDU-Grundkarte läBt sich das "Distanzprofil" (der Grad des Abstands von der standardsprachlichen Idealnorm) zunächst für jeden Ort, dann auch für ganze Gegenden erkennen. Im Blick auf den Gesamtraum wird dieses Distanzprofil „überraschend deutlich von großlandschaftlichen Unterschieden" geprägt:

45 Eichhoft (1977, S. 11). 
„Unūbersehbar ist das generell höhere Abweichungsniveau des Westens gegenüber dem Osten, noch ausgeprägter sind die starken Abweichungen des mitteldeutschen Südens (Zone E), vor allem im Vergleich mit den Zonen $B$ und $C$ im aktuellen bzw. historischen Geltungsbereich niederdeutscher Mundarten. Innerhalb des niederdeutschen Sprachraums ist ein Ansteigen des Abweichungsniveans wiederum nach Norden hin (Mecklenburg, Schleswig-Holstein, Ostfriesland) offenkundig." (S. 122)

Auffallig ist, daß innerhalb des niederdeutschen Sprachraums der Osten eine geringere lexikalische Distanz zum Standarddeutschen aufweist als der Westen. Diese geringere Distanz stellt Müller auch im südlich anschließenden ostmitteldeutschen Raum fest und untermauert damit die schon von Goossens ${ }^{46}$ aufgestellte These von diesem Gebiet als dem Einfallstor für ostmitteldeutsch-standardsprachliches Wortgut in den westniederdeutschen Raum.

\section{Die moderne Welt in der umgangssprachlichen Wortgeographie}

Die auf die Erfassung geographischer Differenzierungen im Wortschatz angelegten Erhebungen von Kretschmer (1918) und Mitzka/Schmitt (1951-80) erwecken den Eindruck, als müBten die Begriffe, deren Bezeichnungen geographisch variieren, wie diese selbst aus historischen Zeiten auf uns gekommen sein. Keiner der $\mathbf{3 3 2}$ mehr oder weniger ausführlichen Wortartikel Kretschmers behandelt moderne Wörter.

Bei der Zusammenstellung des Fragebogens für den WDU wurde Wert darauf gelegt, gerade auch modernes Sachgut zu berücksichtigen, wenn sich irgend Anzeichen für geographische Bezeichnungsgliederung fanden. Das Ergebnis sind insgesamt 17 Wortkarten:

'der Traktor' (Karte 1-12)

'der Negerkuß (Gebäck)' (Karte 2-62)

'Getränk, gemischt aus Bier und Limonade' (Karte 2-73, revidiert als Karte 4-40)

'das Plastik' (Karte 2-77)

'die Blue Jeans' (Karte 2-86)

'der Radfahrer' (Karte 3-17)

'Fußball spielen' (Karte 3-30)

'der Führerschein' (Karte 3-48)

'der Aufzug' (Karte 4-4)

'das Brathähnchen' (Karte 4-36)

'das Brathähnchen (scherzhaft)' (Karte 4-37)

46 Siehe Abschnitt 6.1 . 
'die Limonade' (Karte 4-38)

'das Erfrischungsgetränk aus Cola und Limonade' (Karte 4-39)

'Mischgetränke mit Bier' (Karte 4-41)

'Mischgetränke mit Wein oder Apfelwein' (Karte 4-42)

'der Muckefuck' (Karte 4-43)

'die Pampelmuse' (Karte 4-52)

Neun dieser Begriffe werden im folgenden unter zwei Fragestellungen erörtert: (1), Die Rolle von Amts-, Fach- und Sondersprachen bei der Genese geographischer Differenzierungen im neuzeitlichen Wortschatz, und (2), Sprachschöpfung als Quelle regionalen Wortgebrauchs. ${ }^{47}$

Vier Begriffe verdanken ihre Existenz der modernen Verwaltung und Technik: der Führerschein, der Aufzug, der Traktor und das Plastik. Die zugehörigen Bezeichnungen sind überwiegend künstliche Bildungen oder Entlehnungen aus dem Englischen. Sie dürften zunächst einem geschrieben-fachsprachlichen Bereich angehört haben, aus dem sie dann in die gesprochenen Umgangssprachen gelangt sind. Bezeichnungen für das Dokument, das zum Führen eines Kraftfahrzeug berechtigt, wurden von Behörden auf nationalstaatlicher Ebene festgelegt. Die Bezeichnungsgrenzen fallen deshalb mit nationalen oder administrativen Grenzen zusammen. Die alte Bundesrepublik und Österreich behielten in der Nachkriegszeit die Bezeichnung Führerschein bei. In der DDR war zwecks Meidung des politisch verfemten Bestimmungswortes die Bezeichnung Fahrerlaubnis eingeführt worden. Unsere Wortkarte zeigt im wesentlichen diesen Zustand. Die Führerschein-Meldungen aus diesem Gebiet sind teils Zeugnisse für das inoffizielle Weiterbestehen der Bezeichnung, teils das Ergebnis eines Verwaltungsaktes aus dem Jahr 1982, durch den die Bezeichnung Führerschein auch in der DDR wieder eingeführt wurde. In der deutschsprachigen Schweiz ist Führerausweis die amtliche Bezeichnung, im Wallis allerdings Permis im Einklang mit der französischsprachigen Verwaltung dieses Kantons. In Südtirol gilt Patent, die Übersetzung von patento aus der ursprünglich ganz italienischen Amtssprache.

Neben der amtlichen Bezeichnung gibt es in der Schweiz die in unseren Abfragungen sogar häufiger belegte Variante Fahrausweis. Sie wird mir von Gewährspersonen als "inoffizielle Zweitform" beschrieben - offensichtlich eine Schöpfung der "Umgangssprache" (wiewohl hier in der Form des Dialekts), die kurze und bildhafte Ausdrücke bevorzugt.

$\overline{47}$ Vgl. zum Folgenden z.T. schon Eichhoff (1980). 
Nur zufällig sind auf die Frage nach dem Dokument scherzhafte Bezeichnungen eingegangen, besonders häufig aus der DDR, wo die fast ausschlieBlich indirekte Befragung manche Gewährsperson zu längerem Nachdenken veranlaft haben mag. Nicht weniger als $16 \mathrm{mal}$ wird hier die Fleppen (im Süden die Fleppe) gemeldet. Im Westen liegt Lappen auf derselben sprachlichen Ebene, ist aber nur neunmal gemeldet worden.

Für den Aufzug ist das Lehnwort Lift die älteste Bezeichnung; sie ist mit der Sache aus dem englischen Sprachgebiet gekommen. Lift gilt heute uneingeschränkt in der Schweiz, überwiegt in Österreich und streut im übrigen Gebiet, vor allem in Bayern. Aufzug, die Übersetzung des älteren Fremdwortes, dürfte ursprünglich (wie auch heute noch) fachsprachlich gewesen sein. In den Umgangssprachen ist das Wort im Westmittelund Süddeutschen verbreitet und streut in Österreich. Es zieht sich sich aber auch entlang der Ems bis zur Nordsee. Im übrigen norddeutschen Raum und im gesamten Osten gilt Fahrstuhl. Bei dieser eigenständigen, bildhaften Bezeichnung haben wir es wieder mit einer Schöpfung der Umgangssprache zu tun, die von Hamburg oder Berlin ihren Ausgang genommen haben dürfte.

Die Bezeichnung Traktor für die landwirtschaftliche Zugmaschine ist unter späterer Angleichung an die deutsche Rechtschreibung um die Wende zum 20. Jahrhundert ebenfalls aus dem Englischen entlehnt. Sie gilt uneingeschränkt nur in Österreich, in Südtirol und, mit der Betonung auf der zweiten Silbe, in der Schweiz. Streumeldungen finden sich im Südwesten den Rhein hinab sowie, stärker, in der DDR, letzteres als Folge politisch motivierten Amtsgebrauchs. ${ }^{48}$ Das übrige norddeutsche Gebiet hat die Bezeichnung Trecker, die wohl nicht eine Übersetzung ins Niederdeutsche darstellt, sondern eine norddeutsch-umgangssprachliche, volksetymologische Umbildung des zunächst mündlich übernommenen, lautähnlichen englischen Wortes. ${ }^{49}$ Die im süddeutschen Raum vorherrschende Bezeichnung Bulldog ist trotz ihrer Bildhaftigkeit eine Schöpfung der Industrie. Unter diesem Namen brachte die Firma Lanz in Mannheim im Jahre 1921 ihren Rohöltraktor auf den Markt. Bereits 1925 tritt der ursprüngliche Markenname für landwirtschaftliche Zugmaschinen verallgemeinert auf.

Für den Kunststoff wurde nach dem Krieg in der Bundesrepublik die auf engl. plastics beruhende Bildung (der, später das) Plastik eingeführt. In

48 Eichhoff (1980, S. 159).

49 Aussprache ['trckter]. Man vergleiche denselben Vorgang bei Keks aus engl. cakes. 
die Sprache der DDR fand gleichzeitig der Plast, ebenfalls nach dem engl. Wort, aber mit dem Umweg über das Russische Eingang. Die fachsprachlich überwiegend im Plural verwendete Bezeichnung faBte der gemeine Mann als Singular auf. Die Wortkarte zeigt für die gesamte DDR fast ausschlieBlich die Plaste zu einer Zeit, als die Wörterbücher noch auf der Plast bestanden. Erst nach Erscheinen des WDU hat Plaste, f. dann auch allgemein in die Wörterbücher der DDR Eingang gefunden.

Die bisherigen Fälle zusammenfassend ist festzustellen, dab in der modernen Zeit entstandene geographische Synonyme in Amtsstuben und Fachsprachen ihren Ursprung haben. Da es sich um Begriffe handelt, die in der modernen Welt eine wichtige Rolle spielen, gingen die Bezeichnungen auch in die Umgangssprachen ein. Jedesmal haben die Umgangssprachen aber zumindest regional entweder durch Modifikation oder durch Neuschöpfung eigene Bezeichnungen hervorgebracht.

Zu der Gruppe mit Bezeichnungen amtlichen oder fachsprachlichen Ursprungs gehört der Begriff 'Brathähnchen' nur insofern, als die in der DDR übliche Bezeichnung Broiler aus dem Amerikanischen Englisch in die Fachsprache der industriellen Hühnermästung gelangte und dann als amtliche Bezeichnung des Handels auch in die Umgangssprachen kam. Im Gegensatz zu den obigen Fällen hat der Volksmund dieses griffige und einprägsame Wort nicht mehr verändert.

Die Bezeichnungen für "die blaue, eng anliegende und mit Nieten beschlagene, aus Amerika zu uns gekommene Hose" (so der Text des Fragebogens für den WDU) stellen sich hierher, weil die älteste der Bezeichnungen, Blue Jeans, ${ }^{50}$ wie Lift, Traktor und Plastik mit der Sache aus dem Englischen gekommen ist. Im Falle von Blue Jeans liegt jedoch keine fachsprachliche Vermittlung vor, sondern das, was Leonard Bloomfield ${ }^{51}$ „intimate borrowing" genannt hat. Dabei handelt es sich um Entlehnung in einer Kontaktsituation, in der zwei Sprachen bei miteinander lebenden und kommunizierenden Gruppen (oft in der Form der Diglossie) existieren. Ein solcher Kontakt hat offenbar während der Nachkriegsjahre im Bereich der amerikanischen Besatzungszone Deutschlands bestanden, wobei auf deutscher Seite speziell Jugendliche Sache und Wort aufgenommen haben. Aus diesem sondersprachlichen Bereich ist die Bezeichnung dann Teil der Umgangssprachen und auch der Standardsprache geworden.

50 Das aus engl. blue jeans entlehnte Wort wurde zunächst auf dem zweiten, spāter, wie im amerikanischen Englisch, auf der ersten Silbe betont und zusammen geschrieben.

51 Bloomfield (1933, S. 461-75). 
Die inzwischen historische Karte zeigt Blue Jeans oder Jeans im gesamten Südteil des Sprachgebiets. Außerhalb der amerikanischen Besatzungszone gilt dagegen im Norden Nietenhose, auf dem Gebiet der DDR Niethose, zwei Lehnschöpfungen also, die unabhängig von der englischen Bezeichnung deskriptiv von der Sache ausgehen und daher wahrscheinlich umgangssprachliche Bildungen sind. Der Vollständigkeit halber wäre noch Levis zu nennen, deutlich ein Produkt des Gegenteils von nintimate borrowing", nämlich "cultural borrowing", ${ }^{52}$ dessen Medium das bedruckte Papier ist. Nur so ist die im Deutschen übliche Aussprache ['løVIS] zu erklären, die kein Amerikaner erkennt, weil seine Aussprache ['li:varZ] lautet.

Das Vermögen, schöpferisch mit Sprache umzugehen, war in der älteren Literatur den Dialekten zugesprochen worden. Es sind aber offenbar nicht die Dialekte per se, als Varietäten, denen diese Fähigkeit zukommt, sondern sie findet sich in jeder in lebendigem Gebrauch stehenden Varietät. Gerda Grober-Glück (1975) hat das am Beispiel der Berliner Umgangssprache eindrucksvoll gezeigt. Unter den Karten des WDU sind mehrere mit weiteren Beispielen, wobei sich in fast allen Fällen städtische Umgangssprachen als Quellen erweisen.

Die Mode, Bier mit Limonade zu verdünnen, dürfte um die Jahrhundertwende aus England zu uns gekommen sein, im Zuge der Anglomanie, die uns auch Keks, Beefsteak und Pullover gebracht hat. Die englische Bezeichnung shandygaff wurde nicht mit übernommen. Stattdessen hat jede der drei großen deutschen Städte, in denen die Mode Eingang fand, dem Getränk eine regional motivierte Bezeichnung gegeben. In Hamburg kam man auf Alsterwasser (später auch verkürzt zu Alster) nach dem örtlichen Binnensee, dessen wohl leicht gefärbtes Wasser die Vergleichsbasis abgab. In Berlin entschied sich der Volksmund für Potsdamer, vielleicht zunächst eine Ortsschelte. In München ist die ursprüngliche Bezeichnung (die) Radlermaß (verkürzt der Radler). Die traditionelle Bezeichnung für den einen Liter fassenden Bierkrug wurde also näher definiert durch die süddeutsche Bezeichnung für den Radfahrer. Damit können wir auch den Zeitpunkt der Entstehung der Bezeichnung näher bestimmen. Das Wort Fahrrad kam Ende des 19. Jahrhunderts für Veloziped in Gebrauch. Der erste Beleg für Radlermaß im Archiv des Bairischen Wörterbuchs stammt aus der Zeit um $1920 .^{53}$

52 Bloomfield (1933, S. 444-60).

53 Eichhoff (1980, S. 160). 
Die Wortkarte zeigt, daB sich, offenbar mit der Sache, die Bezeichnung Alsterwasser von Hamburg aus über den Norden, die Bezeichnung Radlermaß über den Süden der alten Bundesrepublik ausgebreitet hatte. Zwischen beiden lag zur Zeit der Erhebung noch eine breite Leerzone. Jedoch berichteten zahlreiche Gewährspersonen am Südrand der Verbreitung von Alsterwasser/Alster und am Nordrand der Verbreitung von Radlermaß/Radler, daB Sache und Bezeichnungen gerade erst aufgekommen seien. Die beiden Bezeichnungen rückten also aufeinander zu mit der Aussicht, gerade im Gebiet der "Mainlinie” aufeinanderzutreffen. Die Sache ist deshalb bei der zweiten Erhebung noch einmal abgefragt worden, und tatsächlich hat sich die Ausbreitung aufeinander zu fortgesetzt. Die zweite Befragung hat aber auch eine andere Tendenz der Umgangssprache ins Licht gerückt: die zum kurzen Ausdruck. Während bei der ersten Abfragung noch fast ausschlieBlich Alsterwasser gemeldet wurde, tritt jetzt daneben deutlich Alster in Erscheinung. Die Form herrscht, wenn ich recht sehe, auch bereits auf den Getränkekarten in Restaurants vor.

$\mathrm{DaB}$ es heute die Umgangssprachen sind und nicht die Mundarten, die die Sprache schöpferisch bereichern, geht aus der kuriosen Eintragung Alsterwater im Hamburgischen Wörterbuch ${ }^{54}$ hervor. Ein Besuch im Archiv des Wörterbuchs ergab, daB es keinen einzigen Beleg für diese Form gab, wohl aber für Alsterwasser, mit dem Vermerk "seit 1926" und „bis jetzt nur hochdeutsch gehört". ${ }^{55}$

Die Wortkarte enthält weitere Belege für volkssprachliche Benennung, darunter Fliegerbier (wohl aus der Kriegszeit, als die Flieger sich beim Alkoholkonsum zurückhalten muBten) und Ententeich (nach der Farbe?).

Neuschöpfung zeigt sich auch bei den Bezeichnungen für das beliebt gewordene Erfrischungsgetränk aus Cola und Limonade. Hier scheint sich die Bezeichnung Spezi durchzusetzen, die den gesamten Süden der Bundesrepublik einnimmt, auch Südtirol, nicht aber die Schweiz und von Österreich nur die westliche Hälfte. Am Ostrand der alten Bundesrepublik zog sich die Bezeichnung bis nach Schleswig-Holstein hinauf, wo sie schon weite Verbreitung gefunden hat.

Der westliche Teil des Sprachgebiets hat zwischen Rhein und Mosel sowie im Ruhrgebiet die Bezeichnung kalter Kaffee, die auch die Ems hinauf gilt und im übrigen im gesamten Norden streut. Zwischen den beiden Gebieten mit kalter Kaffee liegt mit dem Zentrum in der Eifel ein Gebiet mit Diesel, das nordostwärts in Einzelbelegen bis über die

\footnotetext{
54 Scheel (1956-, s.v).

55 Eichhoff (1980, S. 161).
} 
Weser hinweg streut. Um Bremen bis nach Ostfriesland hinein findet sich die Bezeichnung Moorwasser. Alle Bezeichnungen zeigen umgangssprachliches Gepräge. Aufgrund seiner Verbreitung zögert man, als Ausgangspunkt der Bezeichnung Moorwasser Bremen zu vermuten, zumal das Wort eher einer kleinstädtischen Vorstellungswelt entspricht. Kalter Kaffee schmeckt nach Ruhrgebiet, jedoch ist das Gebiet, das in der Pfalz $\mathrm{zwischen}$ Mosel und Rhein besonders kompakt ist, vom Ruhrgebiet weit entfernt. Zudem liegt dazwischen ein Gebiet mit der Bezeichnung Diesel, gewil ebenfalls aufgrund der Farbe so genannt und wiederum ein Bild eher aus der Vorstellungswelt eines modernen Bauern. Man möchte also den vorsichtigen SchluB ziehen, daB auch kleine Städte und das Land als Ausgangspunkt sprachlicher Neuerungen in Betracht zu ziehen sind.

AbschlieBend seien Gummiadler und Flattermann, die scherzhaften Bezeichnungen für das Brathähnchen, genannt. Erstere Bezeichnung war typisch für das Gebiet der DDR sowie für Schleswig-Holstein, streut aber auch im übrigen Bundesgebiet und um die deutsch/schweizerische Grenze. Gummiadler ist seltener und beschränkt sich fast ganz auf Orte nördlich der Mainlinie. Besonders häufig ist das Wort aus dem Ruhrgebiet gemeldet. $\mathrm{Da}$ hier auch andere Bildungen auf - mann beheimatet sind, dürfte die Bezeichnung hier ihren Ursprung haben. Sollte Flattermann eine DDR-typische Bildung sein, fallt es schwer, seine Verbreitung in der alten Bundesrepublik zu erklären.

\section{Schlußbemerkung}

In den fast zwanzig Jahren seit Erscheinen des ersten Bandes des "Wortatlas der deutschen Umgangssprachen" ist das darin veröffentlichte Sprachmaterial in vielfaltiger Weise übernommen und ausgewertet worden: in Wörterbüchern, Enzyklopädien, Schulbüchern, sogar Kochbüchern und natürlich wissenschaftlichen Untersuchungen. Wichtiger noch sind die methodischen Anregungen. die von dem Werk ausgegangen sind. In dem leider noch unveröffentlichten "Wortatlas zur städtischen Umgangssprache der DDR" von Helmut Protze hat er seine erste großräumige Nachfolge gefunden. Einen entschiedenen Schritt weiter geht Heinrich J. Dingeldein mit seinen "Studien zur Wortgeographie der städtischen Alltagssprache in Hessen", einem Werk, in dem die Mehrdimensionalität des alltäglichen Sprachgeschehens zum Gegenstand der Analyse gemacht wird und in dessen Kartenteil es zum ersten Mal wirklich gelingt, durch graphische Mittel die Mehrdimensionalität auf der Sprachkarte sichtbar zu machen. 
Durch den Zugriff auf die Umgangssprachen hat die Areallinguistik nicht nur an Relevanz gewonnen, sondern sich auch ein auf Dauer fruchtbares Arbeitsfeld gesichert.

\section{Literatur}

Besch, Werner/Knoop, Ulrich/Putschke, Wolfgang/Wiegand, Herbert Ernst (Hg.) (1983): Dialektologie. Ein Handbuch zur deutschen und allgemeinen Dialektforschung. Berlin/New York.

Bichel, Ulf (1973): Problem und Begrift der Umgangssprache in der germanistischen Forschung. Tübingen. (Hermaea, 32).

Bloomfield, Leonard (1933): Language. New York.

Deutscher Wortatlas: s. Mitzka/Schmitt (1951-80).

Dingeldein, Heinrich J. (1991): Studien zur Wortgeographie der städtischen Alltagssprache in Hessen. Areale, stratische und diachron-kontrastive Analysen. Tübingen. (Hessische Sprachatlanten, Kleine Reihe, 2).

Duden Deutsches Universalwörterbuch (1989): Hg. [...] unter der Leitung von Günther Drosdowski. 2. Aufl. Mannheim/Wien/Zürich.

Durrell, Martin (1989): Die "Mainlinie” als sprachliche Grenze. In: Dialektgeographie und Dialektologie. Günter Bellmann zum 60. Geburtstag von seinen Schülern und Freunden. Hg. von Wolfgang Putschke/Werner Veith/Peter Wiesinger. Marburg, S. 89-109.

Eichhoff, Jürgen (1972): Geographische Varianten des deutschen Wortschatzes im Untericht der Mittel- und Oberstufe. In: Die Unterrrichtspraxis 5, S. 111-26.

Eichhoff, Jürgen (1976): Zu den Unterschieden und Differenzierungstendenzen im "neutralen" Wortbestand der Bundesrepublik und der DDR. In: Akten des V. Internationalen Germanisten-Kongresses; Cambridge 1975 (Jahrbuch für Internationale Germanistik, Serie A, Bd. II, Nr. 2), S. 95-104.

Eichhoff, Jürgen (1977): Wortatlas der deutschen Umgangssprachen. Bd.1. Bern/München.

Eichhoff, Jürgen (1978): Wortatlas der deutschen Umgangssprachen. Bd.2. Bern/München.

Eichhoff, Jürgen (1980): $\mathrm{Zu}$ einigen im 20. Jahrhundert entstandenen geographischen Unterschieden des Wortgebrauchs in der deutschen Sprache. In: Hildebrandt, Reiner/Friebertshäuser, Hans (Hg.): Sprache und Brauchtum. Bernhard Martin zum 90. Geburtstag. Marburg. (Deutsche Dialektgeographie, 100). S. 154-78.

Eichhoff, Jürgen (1988): Die Wertung landschaftlicher Bezeichnungsvarianten in der deutschen Standardsprache. In: Deutscher Wortschatz. Lexikologische Studien. Ludwig Erich Schmitt zum 80. Geburtstag von seinen Marburger Schülern. Hg. von Horst Haider Munske/Peter von Polenz/Oskar Reichmann/Reiner Hildebrandt. Berlin/New York, S. 511-24.

Eichhoff, Jürgen (1993): Wortatlas der deutschen Umgangssprachen. Bd.3. München/New Providence/London/Paris. 
Friebertshäuser, Hans/Dingeldein, Heinrich J. (1985): Wortgeographie der städtischen Alltagssprache. Ein Forschungsprojekt zum aktuellen Sprachgebrauch in Hessen. In: Zeitschrift für Dialektologie und Linguistik 52, S. 43-52.

Goossens, Jan (1979): Zum Verhätnis von mundartlichem und umgangssprachlichem Wortschatz in Niederdeutschland. In: Gedenkschrift für Heinrich Wesche. Hg. von Wolfgang Kramer/Ulrich Scheuermann/Dieter Stellmacher. Neumünster, S. 39-51.

Grober-Glück, Gerda (1975): Berlin als Innovationszentrum von metaphorischen Wendungen der Umgangssprache. In: Zeitschrift für deutsche Philologie 94, S. 321-67.

Henzen, Walter (1954): Schriftsprache und Mundarten. Ein Überblick über ihr Verhältnis und ihre Zwischenstufen im Deutschen. 2. Aufl. Bern.

Hildebrandt, Reiner (1983): Typologie der arealen lexikalischen Gliederung deutscher Dialekte aufgrund des Deutschen Wortatlasses. In: Besch/Knoop/ Putschke/Wiegand (1983), S. 1331-67.

Hoffmann, Fernand (1979): Sprachen in Luxemburg; Sprachwissenschaftliche und literarhistorische Beschreibung einer Triglossie-Situation. Wiesbaden. (Deutsche Sprache in Europa und Übersee, 6).

Hotzenköcherle, Rudolf (Hg.) (1962-): Sprachatlas der deutschen Schweiz. Bd.1- (bisher 7 Bde). Bern.

Kallmeyer, Werner, Hg. (1994): Kommunikation in der Stadt. Exemplarische Analysen des Sprachverhaltens in Mannheim. 4 Teile. Berlin/New York (Schriften des Instituts für deutsche Sprache, 4.1-4).

Kretschmer, Paul (1918): Wortgeographie der hochdeutschen Umgangssprache. Göttingen. (Nachdruck 1969).

Mangold, Max (1990): Duden Aussprachewörterbuch: Wörterbuch der deutschen Standardaussprache. 3. Aufl. Mannheim/Leipzig/Wien/Zürich.

Mitzka, Walther/(ab Bd.5) Schmitt, Ludwig Erich (Hg.) (1951-80): Deutscher Wortatlas. 22 Bde. Gießen.

Moser, Hugo (1960): „Umgangssprache”. Überlegungen zu ihren Formen und ihrer Stellung im Sprachganzen. In: Zeitschrift für Mundartforschung 27, S. 215-32.

Müller, Gunter (1980): Hochsprachliche lexikalische Norm und umgangssprachlicher Wortschatz im nördlichen Teil Deutschlands. In: Niederdeutsches Wort 20, S. 111-30.

Munske, Horst Haider (1983): Umgangssprache als Sprachkontakterscheinung. In: Besch/Knoop/Putschke/Wiegand (1983), S. 1002-18.

Niebaum, Hermann (1984): Die lexikalische Behandlung des landschaftsgebundenen Wortschatzes in den Wörterbüchern der deutschen Gegenwartssprache. In: Germanistische Linguistik 1-3/83. Studien zur neuhochdeutschen Lexikographie IV. Hildesheim. S. 310-60.

Protze, Helmut (1990): Wortatlas zur städtischen Umgangssprache der DDR. In: Grosse, Rudolf (Hg.): Sprache in der sozialen und kulturellen Entwick- 
lung. Beitrãge eines Kolloquiums zu Ehren von Theodor Frings. Berlin, S. 134-49.

Radtke, Ingulf (1973): Die Umgangssprache. Ein weiterhin ungeklärtes Problem der Sprach wissenschaft. In: Muttersprache 83, S. 161-71.

Rossipal, Hans (1972): Kretschmers „Wortgeographie der hochdeutschen Umgangssprache" aus heutiger Sicht. In: Moderna Sprảk 66, S. 243-58.

Scheel, Käthe (1956-): Hamburgisches Wörterbuch. Hamburg.

Scheuringer, Hermann (1995): Regionale Variation im Deutschen als Dialektalitätsgeographie. In: Dialectologica et Geolinguistica 3 (im Druck).

Sprachatlas der deutschen Schweiz: s. Hotzenköcherle (1962-).

Wahrig, Gerhard (1970): Deutsches Wörterbuch. Gütersloh. (Ungekürzte Sonderausgabe von Gerhard Wahrig: Das große deutsche Wörterbuch. Gütersloh 1966).

Wiesinger, Peter (1980): „Sprache”, „Dialekt” und „Mundart” als sachliches und terminologisches Problem. In: Dialekt und Dialektologie. Ergebnisse des internationalen Symposions "Zur Theorie des Dialekts”. Hg. von Joachim Göschel/Pavle Iviù/Kurt Kehr. Wiesbaden (Zeitschrift für Dialektologie und Linguistik, Beihefte, N.F., 26).

Wiesinger, Peter (1989): The Central and Southern Bavarian Dialects in Bavaria and Austria. In: Russ, Charles V.J. (Hg.): The Dialects of Modern German; A Linguistic Survey. Stanford, S. 438-519.

Zabrotzki, Ludwik (1970): Kommunikative Gemeinschaften und Sprachgemeinschaften. In: Folia Linguistica 4, S. 2-23. 